\title{
Spectroscopic parameters for solar-type stars with moderate-to-high rotation
}

\section{New parameters for ten planet hosts ${ }^{\star} \star \star$}

\author{
M. Tsantaki ${ }^{1,2}$, S. G. Sousa ${ }^{1,2}$, N. C. Santos ${ }^{1,2}$, M. Montalto ${ }^{1}$, E. Delgado-Mena ${ }^{1}$, A. Mortier ${ }^{1}$, \\ V. Adibekyan ${ }^{1}$, and G. Israelian ${ }^{3}$
}

${ }^{1}$ Centro de Astrofísica, Universidade do Porto, Rua das Estrelas, 4150-762 Porto, Portugal e-mail: Maria.Tsantaki@astro.up.pt

2 Departamento de Física e Astronomia, Faculdade de Ciências, Universidade do Porto, Rua do Campo Alegre, 4169-007 Porto, Portugal

3 Instituto de Astrofísica de Canarias, 38200 La Laguna, Tenerife, Spain

Received 22 May 2014 / Accepted 25 July 2014

\begin{abstract}
Context. Planetary studies demand precise and accurate stellar parameters as input for inferring the planetary properties. Different methods often provide different results that could lead to biases in the planetary parameters.

Aims. In this work, we present a refinement of the spectral synthesis technique designed to treat fast rotating stars better. This method is used to derive precise stellar parameters, namely effective temperature, surface gravity, metallicity, and rotational velocity. The procedure is tested for FGK stars with low and moderate-to-high rotation rates.

Methods. The spectroscopic analysis is based on the spectral synthesis package Spectroscopy Made Easy (SME), which assumes Kurucz model atmospheres in LTE. The line list where the synthesis is conducted is comprised of iron lines, and the atomic data are derived after solar calibration.

Results. The comparison of our stellar parameters shows good agreement with literature values, both for slowly and for fast rotating stars. In addition, our results are on the same scale as the parameters derived from the iron ionization and excitation method presented in our previous works. We present new atmospheric parameters for 10 transiting planet hosts as an update to the SWEET-Cat catalog. We also re-analyze their transit light curves to derive new updated planetary properties.
\end{abstract}

Key words. techniques: spectroscopic - stars: fundamental parameters - planets and satellites: fundamental parameters

\section{Introduction}

Since the first discoveries of the extrasolar planets, it has become clear that the derivation of their fundamental properties was directly linked to the properties of their host stars. Until recently, the discovery of extrasolar planets has been substantially fed by the radial velocity (RV) technique. In the past few years, several space missions, such as CoRoT (Baglin et al. 2006) and Kepler (Borucki et al. 2010), as well as ground-based surveys, such as WASP (Pollacco et al. 2006) and HAT-P (Bakos et al. 2004) have successfully used the transit technique. The large number of planets discovered today ${ }^{1}$, allows the study of correlations in the properties of planets and their parent stars, by providing strong

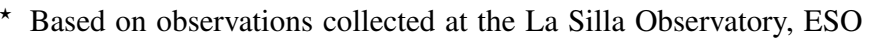
(Chile) with the FEROS spectrograph at the $2.2 \mathrm{~m}$ telescope (ESO runs ID 089.C-0444(A), 088.C-0892(A)) and with the HARPS spectrograph at the $3.6 \mathrm{~m}$ telescope (ESO runs ID 072.C-0488(E), 079.C-0127(A)); at the Observatoire de Haute-Provence (OHP, CNRS/OAMP), France, with the SOPHIE spectrograph at the $1.93 \mathrm{~m}$ telescope and at the Observatoire Midi-Pyrénées (CNRS), France, with the NARVAL spectrograph at the $2 \mathrm{~m}$ Bernard Lyot Telescope (Run ID L131N11).

$\star \star$ Appendix $\mathrm{A}$ is available in electronic form at http://www . aanda.org

1 More than 1800 planets have been discovered to date according to the online catalog: http://www . exoplanet. eu
}

observational constraints on the theories of planet formation and evolution (Mordasini et al. 2012, and references therein).

To understand the physical processes involved in the formation and evolution of planetary systems, precise measurements of the fundamental properties of the exoplanets and their hosts are required. From the analysis of the light curve of a transiting planet, the planetary radius always depends on the stellar radius $\left(R_{\mathrm{p}} \propto R_{\star}\right)$. Moreover, the mass of the planet, or the minimum mass in case the inclination of the orbit is not known, is calculated from the RV curve only if the mass of the star is known $\left(M_{\mathrm{p}} \propto M_{\star}^{2 / 3}\right)$. The fundamental stellar parameters of mass and radius, on the other hand, depend on observationally determined parameters such as, effective temperature $\left(T_{\text {eff }}\right)$, surface gravity $(\log g)$, and metallicity $([\mathrm{Fe} / \mathrm{H}]$, where iron is usually used as a proxy). The last of these fundamental parameters are used to deduce stellar mass and radius either from calibrations (Torres et al. 2010; Santos et al. 2013) or from stellar evolution models (e.g., Girardi et al. 2002).

It is, therefore, imperative to derive precise and accurate stellar parameters to avoid the propagation of errors in the planetary properties. For instance, Torres et al. (2012) have shown that unconstrained parameter determinations derived from spectral synthesis techniques introduce considerable systematic errors in the planetary mass and radius. In particular, residual biases of the stellar radius may explain part of the anomalously inflated 
radii that have been observed for some Jovian planets, such as in the cases of HD 209458 b (Burrows et al. 2000) and WASP-12 b (Hebb et al. 2009).

There are several teams applying different analysis techniques (e.g., photometric, spectroscopic, interferometric), atomic data, model atmospheres, etc., and their results often yield significant differences (e.g., Torres et al. 2008; Bruntt et al. 2012; Molenda-Żakowicz et al. 2013). These systematic errors are difficult to assess and are usually the main contributors to errors within a study. However, these problems can be mitigated by a uniform analysis that will yield the precision needed. Apart from minimizing the errors of the stellar and planetary parameters, uniformity can enhance the statistical significance of correlations between the presence of planets and the properties of their hosts. For example, an overestimation of the stellar radius has been reported in some samples of Kepler objects of interest (Verner et al. 2011; Everett et al. 2013), which in turn leads to an overestimated planetary radius. In this case, planets are perhaps misclassified in the size range that is likely for rocky Earth-like bodies, affecting the planet occurrence rate of Earth-sized planets around solar-type stars.

The high quality stellar spectra obtained from RV planet search programs (e.g., Sousa et al. 2008, 2011), make spectroscopy a powerful tool for deriving the fundamental parameters in the absence of more direct radius measurements (restricted to limited stars with the interferometric technique or to stars that belong to eclipsing binaries). A typical method of deriving stellar parameters for solar-type stars is based on the excitation and ionization equilibrium by measuring the equivalent widths (EW) of iron lines (hereafter the EW method). This method has successfully been applied to RV targets that are restricted to low rotational velocities $(v \sin i)$ to increase the precision of the RV technique (Bouchy et al. 2001). High rotational velocities also limit the precision of the EW method. Spectral lines are broadened by rotation and therefore neighboring lines become blended, often unable to resolve. Even though the EW is preserved, its correct measurement can be highly problematic.

On the other hand, the transit planet hosts have a wider dispersion in rotation rates when compared to the slowly rotating FGK hosts observed with the RV technique. For stars with moderate-to-high rotation rates, which may be the case for the transit targets, spectral synthesis is required for determining the stellar parameters. This technique yields stellar parameters by fitting the observed spectrum with a synthetic one (e.g., Valenti \& Fischer 2005; Malavolta et al. 2014) or with a library of precomputed synthetic spectra (e.g., Recio-Blanco et al. 2006).

In this paper, we propose a refined approach based on the spectral synthesis technique to derive stellar parameters for slowly rotating stars (Sect. 2), yielding results on the same scale with the homogeneous analysis of our previous works (Sect. 3). Our method is tested for a sample of moderate-to-fast FGK rotators (Sect. 4). We also applied our method to a number of planet hosts and provided new stellar parameters. Moreover, their planetary properties are revised (Sect. 5).

\section{Spectroscopic analysis}

Due to severe blending, measuring the EW of stars with high rotational velocity is very difficult, if not impossible (see Fig. 1). In this paper, we focus on deriving precise and accurate parameters for stars with higher $v \sin i$ using the spectral synthesis technique.

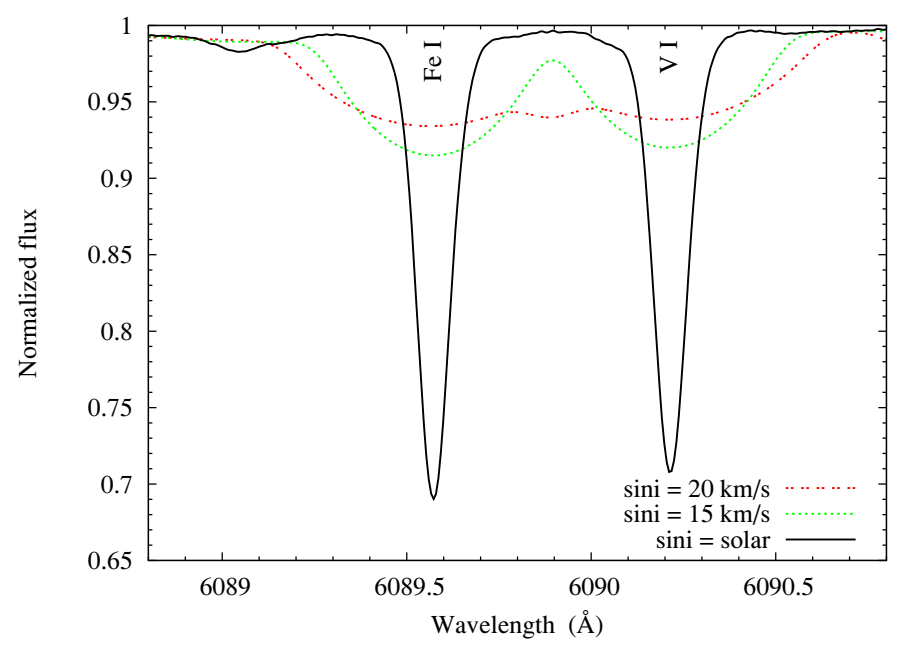

Fig. 1. Solar absorption lines (solid line) broadened by $v \sin i 15 \mathrm{~km} \mathrm{~s}^{-1}$ (green) and $20 \mathrm{~km} \mathrm{~s}^{-1}$ (red). Blending at these rates due to rotation makes accurate measurement of the EW very difficult.

\subsection{Line list}

For an accurate spectral synthesis, atomic and molecular data of all lines in the wavelength intervals where the synthesis is conducted must be as accurate as possible. The choice of intervals for our analysis is based on the line list of iron lines as described in Tsantaki et al. (2013). This list is comprised of weak, isolated iron lines, specifically chosen from the extended line list of Sousa et al. (2008) to exclude blended lines that are commonly found in K-type stars. Effective temperatures derived with this line list agree with the InfraRed-Flux Method (IRFM) for the whole temperature regime of FGK dwarfs.

The spectral window around each iron line is set wide enough to include broadened lines of $v \sin i \sim 50 \mathrm{~km} \mathrm{~s}^{-1}$. Following the Doppler law, such a rotational velocity causes a broadening of $\pm 1 \AA$, around a line in the middle of the optical wavelength range $(\sim 5500 \AA)$.

The original line list contains $137 \mathrm{Fe}$ I and Fe II lines where we set intervals of $2 \AA$ around them. The atomic data for these intervals were obtained from the Vienna Atomic Line Database ${ }^{2}$ (Piskunov et al. 1995; Kupka et al. 1999). We extracted atomic data for all the expected transitions for a star with solar atmospheric parameters for our wavelength intervals. We also included lines predicted for a K-type star with $T_{\text {eff }}=4400 \mathrm{~K}$. The two line lists that correspond to atomic transitions for the two different spectral types were merged into one after removing duplicates. Molecular data of the most abundant molecules in solar-type stars $\left(\mathrm{C}_{2}, \mathrm{CN}, \mathrm{OH}\right.$, and $\left.\mathrm{MgH}\right)$ were also obtained from VALD using the same requests as for the atomic data.

From the above intervals, we selected the optimal ones according to the following procedure. From the first analyses, we noticed that K-type stars show the highest residuals between the observed and the best-fit synthetic spectrum compared to the $\mathrm{F}$ and $\mathrm{G}$ spectral types. The main reason is that the spectra of K-type stars include numerous lines but not all appear in our line list after the requested atomic data queries. Therefore, we discarded lines in the bluer part (below $5000 \AA$ ) where lines are more crowded. Lines within overlapping intervals were merged into one.

In addition, we checked the behavior of the remaining lines due to rotation by using the Sun as a reference star convolved

2 http://vald.inasan.ru/ vald3/php/vald.php 
with moderate rotation of $20 \mathrm{~km} \mathrm{~s}^{-1}$ (see Sect. 4). We excluded lines by eye where there was strong contamination by neighboring lines due to broadening, and chopped the intervals where the contamination in the edges was weak. We also excluded lines that showed high residuals between the spectrum and the synthetic one for the solar parameters.

The initial choice of spectral windows was double the length, i.e. $4 \AA$ where the iron lines were placed in the center. For this length, the best-fit parameters for the Sun (low rotation) were accurate. However, the parameters for the solar spectrum convolved with rotation (again of $20 \mathrm{~km} \mathrm{~s}^{-1}$ ) showed higher deviation $\left(T_{\text {eff }}=5728 \mathrm{~K}, \log g=4.39 \mathrm{dex},[\mathrm{Fe} / \mathrm{H}]=-0.03 \mathrm{dex}\right)$ compared to the standard solar values. For this reason we limited the length of the intervals to $2 \AA$.

Except for blending caused by high rotation, another considerable problem that limits this procedure is the difficulty in distinguishing between the line and the continuum points when the lines become very shallow. In this case, the wings of the lines are miscalculated as continuum, leading to the biases in Sect. 4.

Taking all the above into consideration, the final line list is comprised of $47 \mathrm{Fe}$ I and $4 \mathrm{Fe}$ II lines into 42 wavelength intervals, summing a total of 537 lines of different species. The wavelength intervals and the atomic data of the iron lines are presented in Table $1^{3}$.

Atomic data are usually calculated from laboratory or semiempirical estimates. To avoid uncertainties that may arise from these estimations, we determine astrophysical values for the basic atomic and molecular line data, namely for the oscillator strengths $(\log g f)$ and the van der Waals damping parameters $\left(\Gamma_{6}\right)$. We used the National Solar Observatory Atlas (Kurucz et al. 1984) to improve the transition probabilities and the broadening parameters of our line list in an inverted analysis using fixed the typical solar parameters (as adopted by Valenti \& Fischer 2005): $T_{\text {eff }}=5770 \mathrm{~K}, \log g=4.44 \mathrm{dex},[\mathrm{Fe} / \mathrm{H}]=0.0 \mathrm{dex}$, $v_{\text {mic }}=0.87 \mathrm{~km} \mathrm{~s}^{-1}, v_{\text {mac }}=3.57 \mathrm{~km} \mathrm{~s}^{-1}, \log _{\epsilon}(\mathrm{Fe})=7.50 \mathrm{dex}$.

\subsection{Initial conditions}

All minimization algorithms depend on the initial conditions. To make certain that the convergence is achieved for the global minimum, we set the initial conditions as close to the expected ones as possible. For temperature, we used the calibration of Valenti $\&$ Fischer (2005) as a function of $B-V$ color. Surface gravities were calculated using HIPPARCos parallaxes (van Leeuwen 2007), $V$ magnitudes, bolometric corrections based on Flower (1996) and Torres (2010), and solar magnitudes from Bessell et al. (1998) ${ }^{4}$. When the parallaxes were not available, we used the literature values. Masses were set to the solar value.

Microturbulence $\left(v_{\text {mic }}\right)$ is used to remove possible trends in parameters due to model deficiencies. It has been shown that $v_{\text {mic }}$ correlates mainly with $T_{\text {eff }}$ and $\log g$ for FGK stars (e.g., Nissen 1981; Adibekyan et al. 2012a; Ramírez et al. 2013). We therefore, set $v_{\text {mic }}$ according to the correlation discussed in the work of Tsantaki et al. (2013) for a sample of FGK dwarfs. For the giant stars in our sample, we used the empirical calibration of Mortier et al. (2013a) based on the results of Hekker \& Meléndez (2007).

Macroturbulence $\left(v_{\mathrm{mac}}\right)$ is a broadening mechanism that also correlates with $T_{\text {eff }}$ (e.g., Saar \& Osten 1997). We set $v_{\text {mac }}$ in our analysis following the relation of Valenti \& Fischer (2005).

\footnotetext{
3 The complete line list will be uploaded online in SME format.

4 Surface gravities from parallaxes are usually referred to as trigonometric in the literature.
}

Table 1. Spectral wavelength intervals and line data used for the spectroscopic analysis.

\begin{tabular}{|c|c|c|c|c|c|}
\hline $\begin{array}{c}\text { Intervals } \\
\AA\end{array}$ & $\begin{array}{l}\lambda \\
\AA\end{array}$ & $\mathrm{S}$ & $\begin{array}{l}\chi_{\mathrm{ex}} \\
(\mathrm{eV})\end{array}$ & $\log g f$ & $\Gamma_{6}$ \\
\hline $5521.45-5523.45$ & 5522.45 & $\mathrm{Fe} I$ & 4.21 & -1.484 & -7.167 \\
\hline .11 & 5560.22 & 1 & .43 & .937 & 507 \\
\hline $5632.95-5634.95$ & 5633.95 & $\mathrm{Fe} I$ & .99 & 0.186 & -7.391 \\
\hline $5648.99-5652.47$ & 5649.99 & $\mathrm{Fe} I$ & 5.10 & -0.649 & -7.302 \\
\hline \multirow[t]{2}{*}{$\ldots$} & 5651.47 & $\mathrm{Fe} \mathrm{I}$ & 4.47 & -1.641 & -7.225 \\
\hline & 5652.32 & $\mathrm{Fe} I$ & 4.26 & -1.645 & -7.159 \\
\hline \multirow{2}{*}{$5678.03-5680.97$} & 5679.03 & $\mathrm{Fe} I$ & 4.65 & -0.657 & -7.320 \\
\hline & 5680.24 & $\mathrm{Fe} \mathrm{I}$ & 4.19 & -2.347 & -7.335 \\
\hline 5719 & 5720.90 & $\Gamma$ & & -1.743 & -7.136 \\
\hline $5792.92-5$ & 5793.92 & & & & -7.304 \\
\hline $5810.92-5$ & & & & & \\
\hline \multirow[t]{2}{*}{$5813.81-5815.45$} & & & & & \\
\hline & 58 & & & & \\
\hline \multirow[t]{2}{*}{$5852.15-5854.15$} & 5852. & & & & 201 \\
\hline & 5853.15 & & & & 14 \\
\hline $861.36-58$ & 5862. & & & & 72 \\
\hline $5986.07-5$ & 5987.07 & $\mathrm{Fe}$ & 4.79 & 8 & -7.353 \\
\hline $4.55-6006.55$ & 6005.55 & $\mathrm{~F}$ & 2.59 & -3 . & -7.352 \\
\hline 0.57 & 6089.57 & & -50 & 65 & -7.527 \\
\hline 61 & 6120 & & 2 & -5 & -7.422 \\
\hline $6126.91-6128.78$ & 6127.91 & & 4.14 & -1 & -7.687 \\
\hline .25 & 6149. & Б八 & 3.89 & & -7.478 \\
\hline .62 & 6151 & & 2 & & -7.729 \\
\hline & 6157 & & & & -7.691 \\
\hline & 6173 & & & & -7.829 \\
\hline & 6 & & & & 423 \\
\hline & & & & & \\
\hline & & & & & 59 \\
\hline & & & -2 & & -7 \\
\hline & 6 & & U & & -7 \\
\hline 68 & 6358. & & 6 & 5 & -7.390 \\
\hline .05 & 6432.69 & $\mathrm{Fe} \mathrm{Il}$ & 9 & -3 . & -7.391 \\
\hline & & & 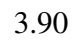 & & -7.682 \\
\hline $6480.88-6$ & 6481.88 & $\mathrm{Fe} I$ & 2.28 & -2.866 & -7.627 \\
\hline $6626.55-6628.55$ & 6627.55 & $\mathrm{Fe} I$ & 4.55 & -1.400 & -7.272 \\
\hline & 6646.94 & $\Gamma_{2}$ & 2.61 & -3.831 & -7.141 \\
\hline 6698. & 6699.15 & $\mathrm{Fe} I$ & 4.59 & -2.004 & -7.162 \\
\hline & & & 4.61 & & 7.539 \\
\hline $6709.32-6$ & 6710.32 & I & & & -7.335 \\
\hline \multirow[t]{2}{*}{ 6724.36-6727.67 } & 6725.36 & $\mathrm{Fe} I$ & 4.10 & & -7.302 \\
\hline & & & & & -7.496 \\
\hline & & & & & -7.130 \\
\hline & 6739.52 & & 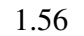 & -4.797 & -7.685 \\
\hline \multirow[t]{2}{*}{ 6744.97-6746.97 } & 6745.11 & $\mathrm{Fe}$ & 4. & -2 & -7.328 \\
\hline & 6745 & & 4.08 & -2.603 & -7.422 \\
\hline .84 & 6839.8 & $\mathrm{Fe} \mathrm{I}$ & 2.56 & -3.304 & -7.567 \\
\hline 67 & 6855.72 & $\mathrm{Fe}$ & 4.61 & -1.885 & -7.253 \\
\hline \multirow[t]{2}{*}{$6856.25-6859.15$} & 6857.25 & $\mathrm{Fe} I$ & 4.08 & -1.996 & -7.422 \\
\hline & 6858. & $\mathrm{Fe} I$ & 4.61 & -0.941 & -7.344 \\
\hline \multirow[t]{2}{*}{$6860.94-6862.94$} & 6861.94 & $\mathrm{Fe} \mathrm{I}$ & 2.42 & -3.712 & -7.580 \\
\hline & 6862.50 & $\mathrm{Fe} \mathrm{I}$ & 4.56 & -1.340 & -7.330 \\
\hline
\end{tabular}

Initial metallicity $([M / H])$ is set to solar and initial rotational velocity to $0.5 \mathrm{~km} \mathrm{~s}^{-1}$.

\subsection{Spectral synthesis}

The spectral synthesis package we use for this analysis is Spectroscopy Made Easy (SME), version 3.3 (Valenti \& Piskunov 1996). Modifications from the first version are described in Valenti et al. (1998) and Valenti \& Fischer (2005). The adopted model atmospheres are generated by the ATLAS9 
program (Kurucz 1993), and local thermodynamic equilibrium is assumed. SME includes the parameter optimization procedure based on the Levenberg-Marquardt algorithm to solve the nonlinear least-squares problem, yielding the parameters that minimize the $\chi^{2}$. In our case, the free parameters are $T_{\text {eff }}, \log g$, $[M / H]$, and $v \sin i$. Metallicity in this work refers to the average abundance of all elements producing absorption in our spectral regions. We can safely assume that $[M / H]$ approximately equals $[\mathrm{Fe} / \mathrm{H}]$ for our sample of stars because the dominant lines in our regions are the iron ones. Additionally, these stars are not very metal-poor $(>-0.58 \mathrm{dex})$. The overall metallicity in metalpoor stars is enhanced by other elements (relative to iron), and in that case the previous assumption does not hold (e.g., Adibekyan et al. 2012b).

After a first iteration with the initial conditions described above, we use the output set of parameters to derive stellar masses using the Padova models ${ }^{5}$ (da Silva et al. 2006). Surface gravity is then re-derived with the obtained mass and temperature. The values of $v_{\text {mic }}$ and $v_{\text {mac }}$ are also updated by the new $T_{\text {eff }}$ and $\log g$. The final results are obtained after a second iteration with the new initial values. Additional iterations were not required, since the results between the first and second iteration in all cases were very close (for instance, the mean differences for the sample in Sect. 4 are $\Delta T_{\text {eff }}=24 \mathrm{~K}, \Delta \log g=0.06 \mathrm{dex}$, $\Delta[\mathrm{Fe} / \mathrm{H}]=0.003 \mathrm{dex}$, and $\Delta v \sin i=0.18 \mathrm{~km} \mathrm{~s}^{-1}$ ).

\subsection{Internal error analysis}

Estimating the errors is a complex problem for this analysis. One approach is to calculate the errors from the covariance matrix of the best-fit solution. Usually these errors are underestimated and include neither deviations depending on the specific choice of initial parameters nor the choice of the wavelength intervals. On the other hand, Monte Carlo approximations are computationally expensive when we are dealing with more than a handful of stars. In this section, we explore the contribution of different type of errors for reference stars of different spectral types. The errors of these stars will be representative of the errors of the whole group that each one belongs to.

We select three slowly rotating stars of different spectral types as our references: F (HD 61421), G (Sun), and K (HD 20868). Their stellar parameters are listed in Table A.1. We convolve each of these stars with different rotational profiles (initial, 15, 25, 35, 45, and $55 \mathrm{~km} \mathrm{~s}^{-1}$ ) to quantitatively check the errors attributed to different $v \sin i$ (see also Sect. 4).

Our aim is to calculate the errors from two different sources: 1) the initial conditions; and 2) the choice of wavelength intervals. First, we check how the initial parameters affect the convergence to the correct ones. For each star we set different initial parameters by changing $T_{\text {eff }} \pm 100 \mathrm{~K}, \log g \pm 0.20 \mathrm{dex}$, $[\mathrm{Fe} / \mathrm{H}] \pm 0.10 \mathrm{dex}$, and $v \sin i \pm 0.50 \mathrm{~km} \mathrm{~s}^{-1}$. We calculate the parameters for the total 81 permutations of this set of initial parameters. This approach is also presented in Valenti \& Fischer (2005) for their solar analysis.

The choice of wavelength intervals is also important for precisely determining of stellar parameters. The spectral window of different instruments varies and therefore, not all wavelength intervals of a specific line list can be used for determining the stellar parameters. Moreover, there are often other reasons for which discarding a wavelength interval would be wise, such as the presence of cosmic rays. In these cases, the errors that are

http://stev. oapd.inaf.it/cgi-bin/param attributed to the discarded wavelength intervals from a defined line list could estimate the homogeneity of our parameters.

We account for such errors by randomly excluding $10 \%$ of our total number of intervals (to leave us with 38 intervals). This percentage is approximately expected for the above cases. Stellar parameters are calculated for the shortened list of intervals, and this procedure is repeated 100 times (each time discarding a random $10 \%$ ). The error of each free parameter is defined as the standard deviation of the results of all repetitions.

For our analysis, we do not include the errors derived from the convariance matrix. The primary reason is that the flux errors of each wavelength element that are required for the precise calculation of the convariance matrix, unfortunately, are not provided for our spectra. In such cases one has to be careful with the interpretation of the values of the covariance matrix.

Table 2 shows the errors derived from the two different sources described above. The errors in $T_{\text {eff }}$ and $\log g$ caused by the different initial parameters are slightly more significant, whereas for $[\mathrm{Fe} / \mathrm{H}]$ and $v \sin i$ both type of errors are comparable. Finally, we add quadratically the two sources of errors that are described above (see Table 3 ). We notice that for higher $v \sin i$, the uncertainties in all parameters become higher as one would expect. K-type stars also have higher uncertainties than Fand G-types. In particular, the uncertainties in $v \sin i$, for K-type stars, are significantly high for $v \sin i>45 \mathrm{~km} \mathrm{~s}^{-1}$. Fortunately, $\mathrm{K}$-type stars are typically slow rotators since rotational velocity decreases with the spectral type for FGK stars (e.g., Gray 1984; Nielsen et al. 2013).

\section{Spectroscopic parameters for slowly rotating FGK stars}

To test the effectiveness of the line list, we used a sample of 48 FGK stars (40 dwarfs and 8 giants) with low rotation rates, high signal-to-noise ratio $(\mathrm{S} / \mathrm{N})$, and high resolution spectra, most of them taken from the archival data of HARPS $(R \sim$ $110000)$ and the rest with UVES $(R \sim 110000)$, and FEROS $(R \sim 48000)$ spectrographs. Their stellar parameters range from $4758 \leq T_{\text {eff }} \leq 6666 \mathrm{~K}, 2.82 \leq \log g \leq 4.58 \mathrm{dex}$, and $-0.58 \leq$ $[\mathrm{Fe} / \mathrm{H}] \leq 0.33 \mathrm{dex}$, and they are derived following the method described in Sect. 2. Figure 2 depicts the comparison between the parameters derived in this work and the EW method. All parameters from the EW method were taken from Sousa et al. (2011), Mortier et al. (2013a,b), Tsantaki et al. (2013), and Santos et al. (2013) using the same methodology to provide the best possible homogeneity for the comparison. The differences between these methods are presented in Table 4 and the stellar parameters in Table A.1.

The effective temperatures derived with the spectral synthesis technique and the EW method are in good agreement. The greatest discrepancies appear for $T_{\text {eff }}>6000 \mathrm{~K}$, where the effective temperature derived from this work is systematically cooler. The same systematics are also presented in Molenda-Żakowicz et al. (2013), where the authors compare the EW method with other spectral synthesis techniques, but the explanation for these discrepancies is not clear yet.

The values of metallicity are in perfect agreement between the two methods.

Surface gravity is a parameter that is the most difficult to constrain with spectroscopy. The comparison of the two methods shows a considerable offset of 0.19 dex, where $\log g$ is underestimated compared to the EW method. Interestingly, this offset is smaller for giant stars $(\Delta \log g=0.07 \mathrm{dex})$ than for dwarfs $(\Delta \log g=-0.24 \mathrm{dex})$. 
M. Tsantaki et al.: Spectroscopic parameters for solar-type starswith moderate-to-high rotation

Table 2. Internal error analysis for each spectral type and different rotational velocities.

\begin{tabular}{|c|c|c|c|c|c|c|c|c|c|c|c|c|}
\hline \multirow{3}{*}{$\begin{array}{l}\text { Parameters } \\
v \sin i\end{array}$} & \multicolumn{12}{|c|}{ F-type (HD 61421) } \\
\hline & \multicolumn{6}{|c|}{ Initial parameters } & \multicolumn{6}{|c|}{ Wavelength choice } \\
\hline & $0 \mathrm{~km} \mathrm{~s}^{-1}$ & $15 \mathrm{~km} \mathrm{~s}^{-1}$ & $25 \mathrm{~km} \mathrm{~s}^{-1}$ & $35 \mathrm{~km} \mathrm{~s}^{-1}$ & $45 \mathrm{~km} \mathrm{~s}^{-1}$ & $55 \mathrm{~km} \mathrm{~s}^{-1}$ & $0 \mathrm{~km} \mathrm{~s}^{-1}$ & $15 \mathrm{~km} \mathrm{~s}^{-1}$ & $25 \mathrm{~km} \mathrm{~s}^{-1}$ & $35 \mathrm{~km} \mathrm{~s}^{-1}$ & $45 \mathrm{~km} \mathrm{~s}^{-1}$ & $55 \mathrm{~km} \mathrm{~s}^{-1}$ \\
\hline$T_{\text {eff }}(\mathrm{K})$ & 27 & 43 & 48 & 54 & 97 & 108 & 13 & 9 & 17 & 48 & 16 & 85 \\
\hline $\log g($ dex $)$ & 0.11 & 0.15 & 0.21 & 0.25 & 0.20 & 0.17 & 0.02 & 0.02 & 0.08 & 0.16 & 0.03 & 0.05 \\
\hline$[\mathrm{Fe} / \mathrm{H}](\mathrm{dex})$ & 0.03 & 0.04 & 0.05 & 0.07 & 0.06 & 0.07 & 0.01 & 0.01 & 0.02 & 0.04 & 0.01 & 0.01 \\
\hline \multirow[t]{3}{*}{$v \sin i\left(\mathrm{~km} \mathrm{~s}^{-1}\right)$} & 0.20 & 0.25 & 0.98 & 1.00 & 1.57 & 2.60 & 0.08 & 0.14 & 0.91 & 0.89 & 0.93 & 2.13 \\
\hline & \multicolumn{12}{|c|}{ G-type (Sun) } \\
\hline & \multicolumn{6}{|c|}{ Initial parameters } & \multicolumn{6}{|c|}{ Wavelength choice } \\
\hline$v \sin i$ & $0 \mathrm{~km} \mathrm{~s}^{-1}$ & $15 \mathrm{~km} \mathrm{~s}^{-1}$ & $25 \mathrm{~km} \mathrm{~s}^{-1}$ & $35 \mathrm{~km} \mathrm{~s}^{-1}$ & $45 \mathrm{~km} \mathrm{~s}^{-1}$ & $55 \mathrm{~km} \mathrm{~s}^{-1}$ & $0 \mathrm{~km} \mathrm{~s}^{-1}$ & $15 \mathrm{~km} \mathrm{~s}^{-1}$ & $25 \mathrm{~km} \mathrm{~s}^{-1}$ & $35 \mathrm{~km} \mathrm{~s}^{-1}$ & $45 \mathrm{~km} \mathrm{~s}^{-1}$ & $55 \mathrm{~km} \mathrm{~s}^{-1}$ \\
\hline$T_{\text {eff }}(\mathrm{K})$ & 12 & 5 & 18 & 86 & 86 & 147 & 13 & 9 & 21 & 36 & 43 & 116 \\
\hline $\log g(\operatorname{dex})$ & 0.06 & 0.05 & 0.09 & 0.16 & 0.11 & 0.14 & 0.01 & 0.03 & 0.07 & 0.07 & 0.07 & 0.12 \\
\hline$[\mathrm{Fe} / \mathrm{H}](\mathrm{dex})$ & 0.02 & 0.01 & 0.04 & 0.07 & 0.07 & 0.07 & 0.02 & 0.01 & 0.03 & 0.02 & 0.02 & 0.04 \\
\hline \multirow[t]{3}{*}{$v \sin i\left(\mathrm{~km} \mathrm{~s}^{-1}\right)$} & 0.25 & 0.09 & 0.32 & 0.36 & 2.47 & 3.67 & 0.11 & 0.99 & 0.26 & 0.17 & 2.29 & 3.49 \\
\hline & \multicolumn{12}{|c|}{ K-type (HD 20868) } \\
\hline & \multicolumn{6}{|c|}{ Initial parameters } & \multicolumn{6}{|c|}{ Wavelength choice } \\
\hline$v \sin i$ & $0 \mathrm{~km} \mathrm{~s}^{-1}$ & $15 \mathrm{~km} \mathrm{~s}^{-1}$ & $25 \mathrm{~km} \mathrm{~s}^{-1}$ & $35 \mathrm{~km} \mathrm{~s}^{-1}$ & $45 \mathrm{~km} \mathrm{~s}^{-1}$ & $55 \mathrm{~km} \mathrm{~s}^{-1}$ & $0 \mathrm{~km} \mathrm{~s}^{-1}$ & $15 \mathrm{~km} \mathrm{~s}^{-1}$ & $25 \mathrm{~km} \mathrm{~s}^{-1}$ & $35 \mathrm{~km} \mathrm{~s}^{-1}$ & $45 \mathrm{~km} \mathrm{~s}^{-1}$ & $55 \mathrm{~km} \mathrm{~s}^{-1}$ \\
\hline$T_{\text {eff }}(\mathrm{K})$ & 20 & 52 & 54 & 77 & 130 & 127 & 15 & 47 & 45 & 68 & 110 & 110 \\
\hline $\log g(\operatorname{dex})$ & 0.09 & 0.16 & 0.17 & 0.20 & 0.14 & 0.18 & 0.03 & 0.11 & 0.12 & 0.16 & 0.10 & 0.09 \\
\hline$[\mathrm{Fe} / \mathrm{H}](\mathrm{dex})$ & 0.02 & 0.06 & 0.09 & 0.09 & 0.09 & 0.09 & 0.02 & 0.05 & 0.07 & 0.07 & 0.02 & 0.01 \\
\hline$v \sin i\left(\mathrm{~km} \mathrm{~s}^{-1}\right)$ & 0.46 & 0.72 & 0.86 & 2.38 & 6.92 & 7.54 & 0.45 & 0.69 & 0.77 & 2.12 & 6.98 & 7.36 \\
\hline
\end{tabular}

Notes. Two sources of errors were considered: initial parameters and wavelength choice (dependence on the choice of the $90 \%$ of the total intervals). Rotational velocity $0 \mathrm{~km} \mathrm{~s}^{-1}$ refers to the intrinsic $v \sin i$ of the star.

Table 3. Errors summed quadratically for each spectral type and for the different rotational velocities.

\begin{tabular}{|c|c|c|c|c|c|c|}
\hline \multirow{2}{*}{$\begin{array}{l}\text { Parameters } \\
v \sin i\end{array}$} & \multicolumn{6}{|c|}{ F-type (HD 61421) } \\
\hline & $0 \mathrm{~km} \mathrm{~s}^{-1}$ & $15 \mathrm{~km} \mathrm{~s}^{-1}$ & $25 \mathrm{~km} \mathrm{~s}^{-1}$ & $35 \mathrm{~km} \mathrm{~s}^{-1}$ & $45 \mathrm{~km} \mathrm{~s}^{-1}$ & $55 \mathrm{~km} \mathrm{~s}^{-1}$ \\
\hline$T_{\text {eff }}(\mathrm{K})$ & 30 & 44 & 51 & 72 & 98 & 137 \\
\hline $\log g($ dex $)$ & 0.11 & 0.15 & 0.22 & 0.30 & 0.20 & 0.18 \\
\hline$[\mathrm{Fe} / \mathrm{H}](\mathrm{dex})$ & 0.03 & 0.04 & 0.05 & 0.03 & 0.08 & 0.06 \\
\hline \multirow[t]{2}{*}{$v \sin i\left(\mathrm{~km} \mathrm{~s}^{-1}\right)$} & 0.22 & 0.29 & 1.34 & 1.34 & 1.82 & 3.36 \\
\hline & \multicolumn{6}{|c|}{ G-type (Sun) } \\
\hline$v \sin i$ & $0 \mathrm{~km} \mathrm{~s}^{-1}$ & $15 \mathrm{~km} \mathrm{~s}^{-1}$ & $25 \mathrm{~km} \mathrm{~s}^{-1}$ & $35 \mathrm{~km} \mathrm{~s}^{-1}$ & $45 \mathrm{~km} \mathrm{~s}^{-1}$ & $55 \mathrm{~km} \mathrm{~s}^{-1}$ \\
\hline$T_{\text {eff }}(\mathrm{K})$ & 18 & 10 & 28 & 93 & 96 & 187 \\
\hline $\log g(\operatorname{dex})$ & 0.06 & 0.06 & 0.11 & 0.17 & 0.13 & 0.18 \\
\hline$[\mathrm{Fe} / \mathrm{H}](\mathrm{dex})$ & 0.03 & 0.01 & 0.05 & 0.07 & 0.07 & 0.08 \\
\hline \multirow[t]{2}{*}{$v \sin i\left(\mathrm{~km} \mathrm{~s}^{-1}\right)$} & 0.27 & 0.99 & 0.41 & 0.40 & 3.37 & 5.06 \\
\hline & \multicolumn{6}{|c|}{ K-type (HD 20868) } \\
\hline$v \sin i$ & $0 \mathrm{~km} \mathrm{~s}^{-1}$ & $15 \mathrm{~km} \mathrm{~s}^{-1}$ & $25 \mathrm{~km} \mathrm{~s}^{-1}$ & $35 \mathrm{~km} \mathrm{~s}^{-1}$ & $45 \mathrm{~km} \mathrm{~s}^{-1}$ & $55 \mathrm{~km} \mathrm{~s}^{-1}$ \\
\hline$T_{\text {eff }}(\mathrm{K})$ & 25 & 70 & 70 & 103 & 170 & 168 \\
\hline $\log g(\mathrm{dex})$ & 0.09 & 0.19 & 0.21 & 0.26 & 0.17 & 0.20 \\
\hline$[\mathrm{Fe} / \mathrm{H}](\mathrm{dex})$ & 0.03 & 0.08 & 0.11 & 0.11 & 0.09 & 0.09 \\
\hline$v \sin i\left(\mathrm{~km} \mathrm{~s}^{-1}\right)$ & 0.64 & 0.99 & 1.15 & 3.19 & 9.83 & 10.54 \\
\hline
\end{tabular}

Table 4. Differences in stellar parameters between this work and the EW method for the 48 sample stars.

\begin{tabular}{ccccc}
\hline \hline This work - EW & $\Delta T_{\text {eff }}{ }^{a}( \pm \mathrm{MAD}) \mathrm{K}$ & $\Delta \log g( \pm \mathrm{MAD}) \mathrm{dex}$ & $\Delta[\mathrm{Fe} / \mathrm{H}]( \pm \mathrm{MAD}) \mathrm{dex}$ & $N$ \\
\hline Whole sample & $-26 \pm 14( \pm 55)$ & $-0.19 \pm 0.04( \pm 0.14)$ & $0.000 \pm 0.010( \pm 0.041)$ & 48 \\
F-type & $-97 \pm 22( \pm 68)$ & $-0.34 \pm 0.07( \pm 0.18)$ & $0.006 \pm 0.014( \pm 0.014)$ & 12 \\
G-type & $7 \pm 16( \pm 36)$ & $-0.07 \pm 0.05( \pm 0.04)$ & $0.019 \pm 0.021( \pm 0.032)$ & 18 \\
K-type & $-5 \pm 27( \pm 32)$ & $-0.18 \pm 0.05( \pm 0.16)$ & $-0.027 \pm 0.016( \pm 0.042)$ & 18 \\
\hline
\end{tabular}

Notes. MAD values correspond to the median average deviation and are indicated in parenthesis. The differences are also listed per spectral type. $\mathrm{N}$ is the number of stars used for the comparison. ${ }^{(a)}$ The standard errors of the mean $\left(\sigma_{M}\right)$ are calculated with the following formula: $\sigma_{M}=\frac{\sigma}{\sqrt{N}}$, with $\sigma$ the standard deviation.

To further investigate these differences, we compared the spectroscopic $\log g$ with surface gravity derived with another method that is less model dependent. For 16 dwarf stars in our sample that have a transiting planet, surface gravity can be derived from the analysis of the transit light curve (see also Sect. 5). We compared $\log g$ derived from the transit light curve 

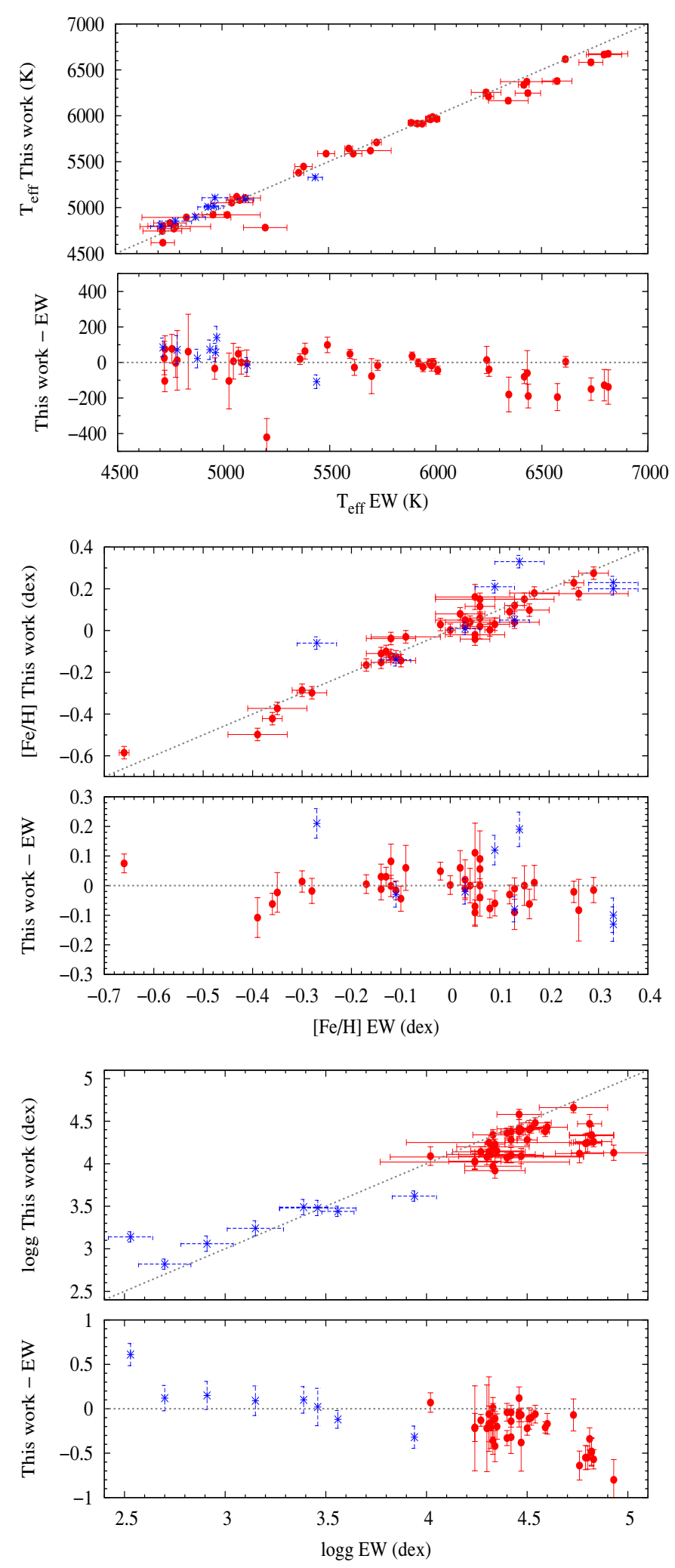

Fig. 2. Comparison between the parameters derived using the spectral synthesis method (This work) and the results of our EW method: temperature (top panel), metallicity (middle panel), and surface gravity (bottom panel). Circles represent dwarf stars and asterisks giants.

with the spectroscopic $\log g$ from the EW method (both values from Mortier et al. 2013b) and this work (see Fig. 3).

We show that $\log g$ from the EW analysis is overestimated for low $\log g$ values and underestimated for high $\log g$ values.
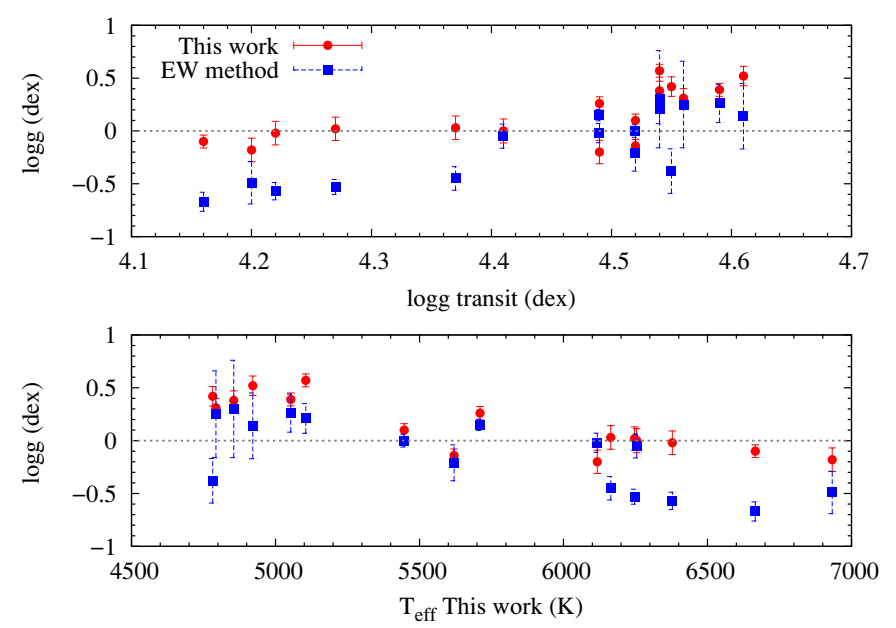

Fig. 3. Comparison of surface gravity derived from the transit fit with this work and the EW method. $\Delta \log g$ represents "transit minus this work" (red circles) and "transit minus EW method" (blue squares).

Fortunately, this trend does not affect $T_{\text {eff }}$ and $[\mathrm{Fe} / \mathrm{H}]$, as shown in the recent work of Torres et al. (2012). The same systematics were also found between the $\log g$ from the EW method and the $\log g$ derived with the HIPPARCos parallaxes for solar-type stars in Tsantaki et al. (2013) and Bensby et al. (2014). These results imply that $\log g$ from the EW method using iron lines suffers from biases, but the explanation is not clear.

On the other hand, $\log g$ derived from this work is in very good agreement with the transit $\log g$, for values lower than 4.5 dex. Stars with $\log g>4.5$ dex correspond to the cooler stars and are also underestimated. The reason for this underestimation is not yet known, so further investigation is required to understand this behavior.

Despite the differences for the $\log g$ values of mainly the F-type stars, the results listed in Table 4 show that for slowly rotating FGK stars, stellar parameters derived from both methods are on the same scale. This means that for the whole sample, the residuals between both methods are small and of the same order of magnitude as the errors of the parameters.

\section{Spectroscopic parameters for fast rotating FGK dwarfs}

Testing our method for slow FGK rotators does not necessary imply that it will work for higher $v \sin i$ where spectral lines are much more broadened and shallower. Our goal is to examine how efficient our method is for stars with moderate-to-high rotation rates. For this purpose, we derived stellar parameters for reference stars of different spectral type and with low $v \sin i$. Second, these stars are convolved with a set of rotational profiles using the rotin3 routine as part of the SYNSPEC synthesis code ${ }^{6}$ (Hubeny et al. 1994). As a result, each star has eight different rotational velocities (initial, 5, 10, 15, 20, 25, 30, 40, $50 \mathrm{~km} \mathrm{~s}^{-1}$ ). Stellar parameters of all rotational profiles were calculated to investigate how they differ from the non-broadened (unconvolved) star. This test is an indication of how the accuracy of our method is affected by adding a rotational profile.

The selected reference stars are two F-type, one G-type, and four K-type stars, and they are presented in boldface in Table A.1. Probably one star per spectral type would be enough,

6 http://nova.astro.umd.edu/Synspec43/ 

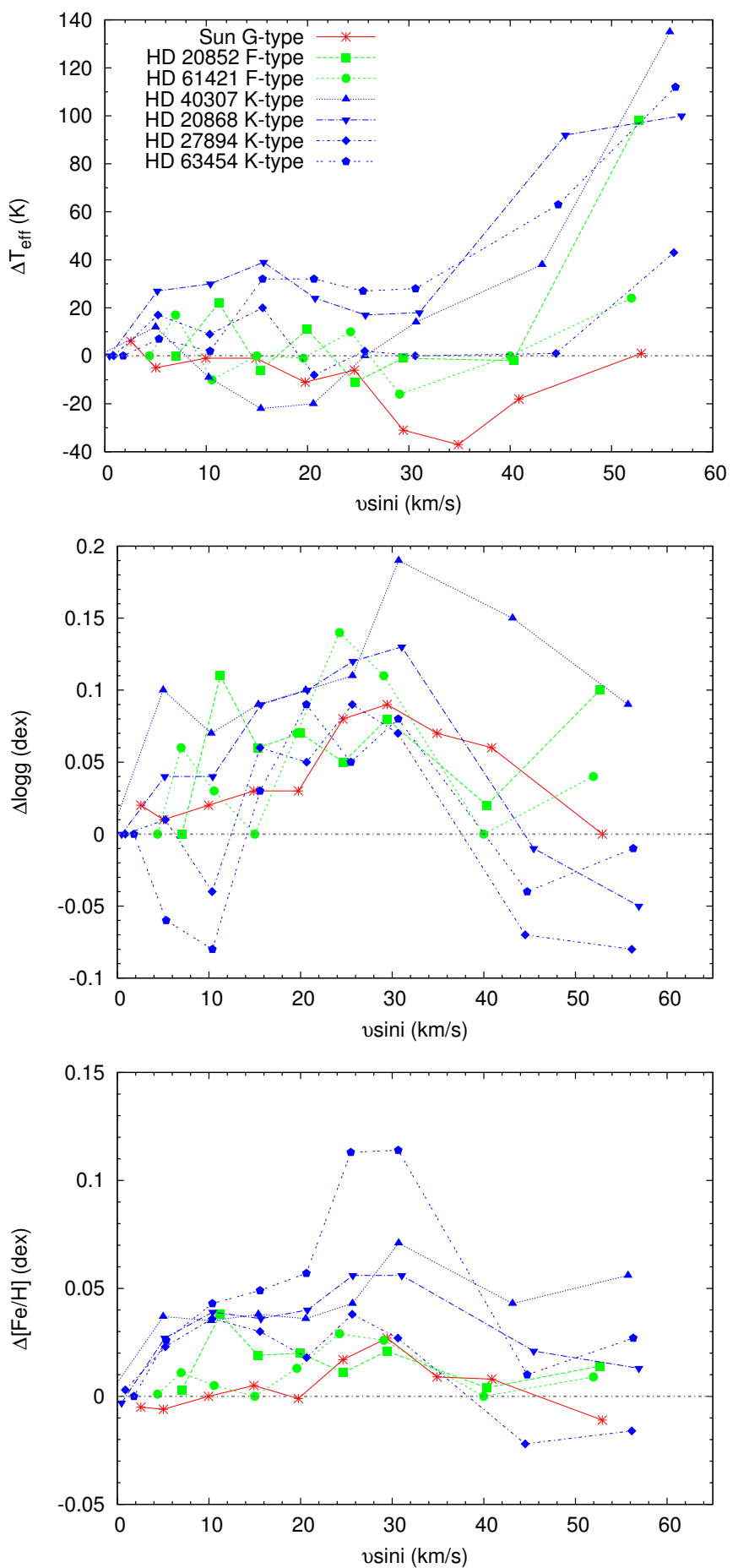

Fig. 4. Differences in temperature (top panel), surface gravity (middle panel), and metallicity (bottom panel; initial $v \sin i$ minus the different rotational profiles) vs. $v \sin i$. Each star is represented by a different symbol, and each spectral type is represented by a different color: blue for K-type, red for G-type, and green F-type. The initial $v \sin i$ is different for each star.

but we included more F- and K-type stars because they showed higher uncertainties (especially the K-type stars). In Fig. 4 the differences of stellar parameters between the stars with the unconvolved values (original $v \sin i$ ), and the convolved ones are plotted for the eight different rotational velocities.

As $v \sin i$ increases, K-type stars show the highest differences in the stellar parameters compared to the non-broadened
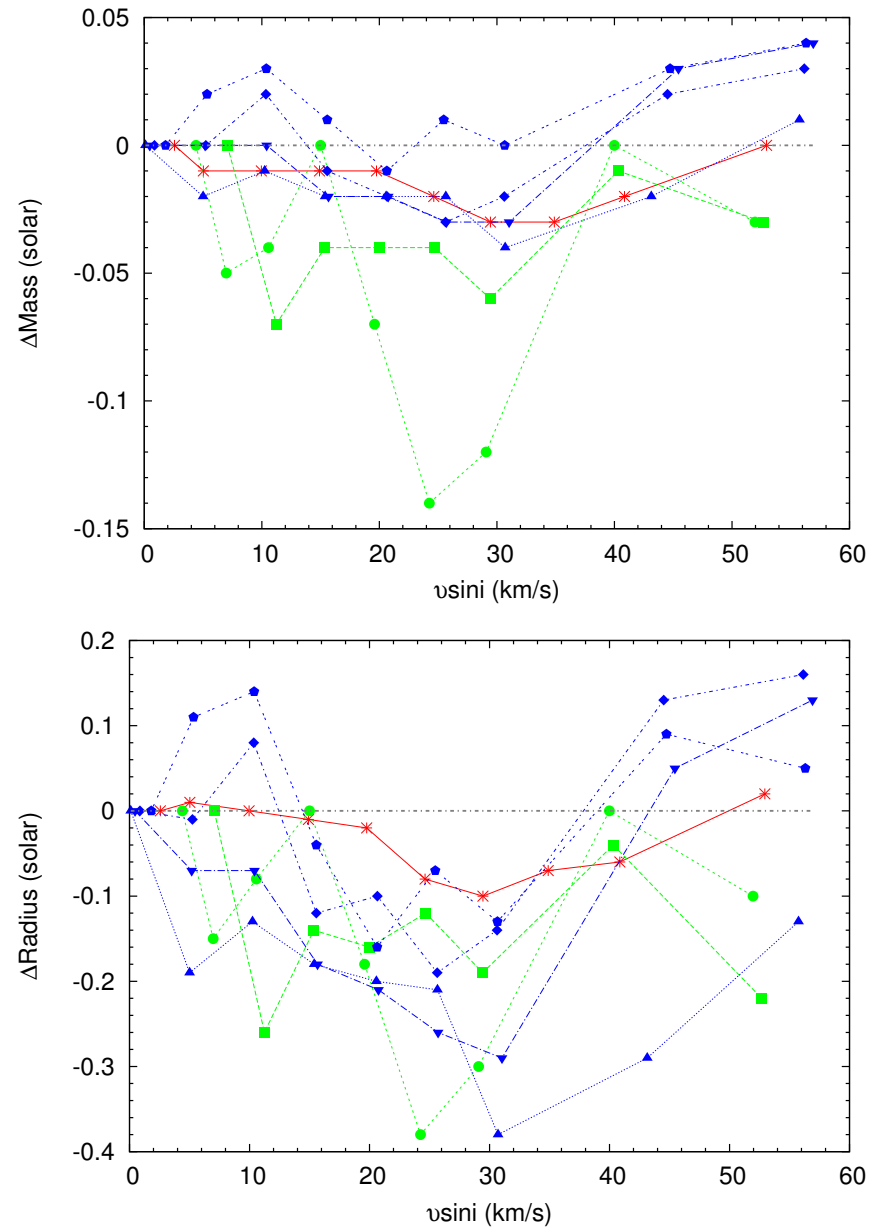

Fig. 5. Differences in stellar mass (top panel) and radius (bottom panel) vs. $v \sin i$. The symbols are the same as in Fig. 4.

profile. These deviations for high $v \sin i$ are also shown in the error analysis of Sect. 2.4. The temperatures of these stars are systematically underestimated with increasing $v \sin i$. On the other hand, the parameters of F- and G-type stars are very close to the ones with low rotation, and no distinct trends are observed with rotation. Even for very high $v \sin i$, temperature and metallicity can be derived with differences in values of less than $100 \mathrm{~K}$ and 0.05 dex respectively. Surface gravity, however, shows large differences that reach up to $\sim 0.20$ dex.

The above discrepancies in the parameters in turn affect the stellar mass and radius. To investigate these offsets, we calculate the mass and radius for all the rotational velocities using the calibration of Torres et al. (2010) but corrected for small offsets to match masses derived from isochrone fits by Santos et al. (2013). The results in Fig. 5 show that the mass hardly changes as $v \sin i$ increases. The stellar radius, however, is affected in the same manner as surface gravity with greater radius differences. For example, the maximum difference in $\log g(\sim 0.20$ dex $)$ causes a deviation in radius of $0.39 R_{\odot}$.

\subsection{Application to FGK fast rotators}

We selected a sample of FGK dwarfs with moderate-to-high $v \sin i$, which have available several estimates of their parameters from different techniques in the literature (see references in Table A.2). From these references, we included 17 stars with $v \sin i$ up to $54 \mathrm{~km} \mathrm{~s}^{-1}$ that have spectra available in 
Table 5. Differences in parameters derived with different methods. $N$ indicates the number of stars used for the comparison.

\begin{tabular}{lcccc}
\hline \hline & $\Delta T_{\text {eff }}(\mathrm{K})$ & $\Delta \log g(\mathrm{dex})$ & $\Delta[\mathrm{Fe} / \mathrm{H}](\mathrm{dex})$ & $N$ \\
\hline \multirow{2}{*}{ This work - EW } & $3 \pm 48$ & $-0.11 \pm 0.07$ & $0.04 \pm 0.03$ & 11 \\
& $(\mathrm{MAD}=80)$ & $(\mathrm{MAD}=0.24)$ & $(\mathrm{MAD}=0.03)$ \\
\multirow{2}{*}{ This work - Synthesis } & $32 \pm 29$ & $0.03 \pm 0.05$ & $0.05 \pm 0.02$ & 29 \\
& $(\mathrm{MAD}=64)$ & $(\mathrm{MAD}=0.15)$ & $(\mathrm{MAD}=0.04)$ \\
\multirow{2}{*}{ This work - Photometry } & $-12 \pm 25$ & $0.06 \pm 0.02$ & - & 18 \\
& $(\mathrm{MAD}=44)$ & $(\mathrm{MAD}=0.04)$ & - & \\
\hline
\end{tabular}

Table 6. Observation log of the transit hosts analyzed in this work. $V$ magnitudes are taken from SIMBAD.

\begin{tabular}{lcccc}
\hline \hline Star & $V(\mathrm{mag})$ & Spectrograph & Resolution & $S / N$ \\
\hline 30 Ari B & 7.09 & FEROS & 48000 & 270 \\
HAT-P-23 & 13.05 & FEROS & 48000 & 65 \\
HAT-P-34 & 10.40 & FEROS & 48000 & 145 \\
HAT-P-41 & 11.36 & FEROS & 48000 & 135 \\
HAT-P-2 & 8.69 & SOPHIE & 75000 & 250 \\
XO-3 & 9.85 & SOPHIE & 75000 & 130 \\
HD 8673 & 6.31 & NARVAL & 75000 & 222 \\
Kepler-410A & 9.50 & NARVAL & 75000 & 72 \\
CoRoT-11 & 12.80 & HARPS & 110000 & 116 \\
CoRoT-3 & 13.29 & HARPS & 110000 & 84 \\
\hline
\end{tabular}

Notes. S/N is estimated at $6070 \AA$.

the public archives of different high resolution instruments (HARPS, FEROS, ELODIE, and CORALIE). The spectra were already processed with their standard pipeline procedures. We corrected for the radial velocity shifts and in cases of multiple observations, the spectra were summed using the IRAF tools, dopcor and scombine.

We derived the stellar parameters with the method in this work, and the results and literature values of the sample are presented in Table A.2. Table 5 shows the differences between the stellar parameters of this work and the different methods used: other spectral synthesis techniques, the EW method (until $v \sin i \sim 10 \mathrm{~km} \mathrm{~s}^{-1}$ ), and the photometric technique, namely IRFM. The differences between this work and other methods are very small for all parameters.

In Fig. 6 we plot the comparison between the literature values and our results. Figure 7 shows each stellar parameter in dependence of rotational velocity for the different methods and for this work. Even though the mean differences in temperature are close to zero, a slight overestimation of our method appears for high $v \sin i$. Surface gravity shows the lowest dispersion when compared to trigonometric $\log g$ from all methods. Metallicity is also in agreement, excluding perhaps an outlier (HD 49933).

\section{Data and spectroscopic parameters for planet hosts}

We have identified spectra for ten confirmed planet hosts that show relatively high $v \sin i$, and we were unable to apply our standard EW method for their spectroscopic analysis. We use the procedure of this work to derive their stellar parameters to update the online SWEET-Cat catalog where stellar parameters for FGK and M planet hosts ${ }^{7}$ are presented (Santos et al. 2013). These stars were observed with high resolution spectrographs (Table 6) gathered by our team (these spectra have never been

\footnotetext{
7 https://www.astro.up.pt/resources/sweet-cat/
}
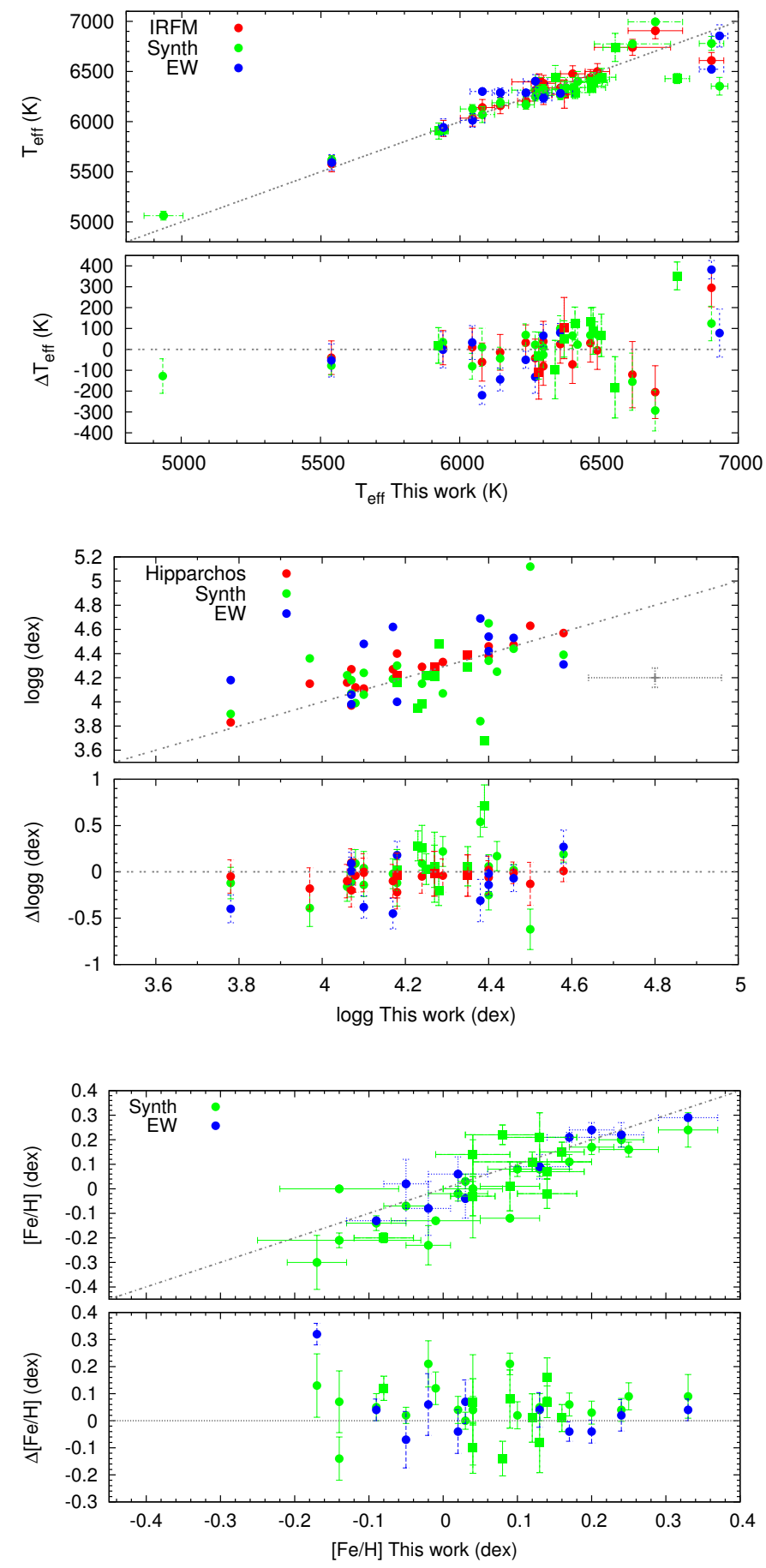

Fig. 6. Temperature (top panel), surface gravity (middle panel), and metallicity (bottom panel). Different colors represent different techniques. Square symbols represent planet hosts analyzed in this work. The average error is plotted in the middle panel. In each panel, the upper plot compares the data and the lower plot the residual differences from perfect agreement.

analyzed before) and by the use of the archive (for the NARVAL spectra). Their spectral type varies from $F$ to $G$.

The spectra were reduced with the standard pipelines and are corrected with the standard IRAF tools for the radial velocity shifts and their spectra are added in cases of multiple exposures of individual observed stars. Following the procedure presented in this work, we derived their fundamental parameters, which are included in Figs. 6 and 7 (square symbols) and presented 

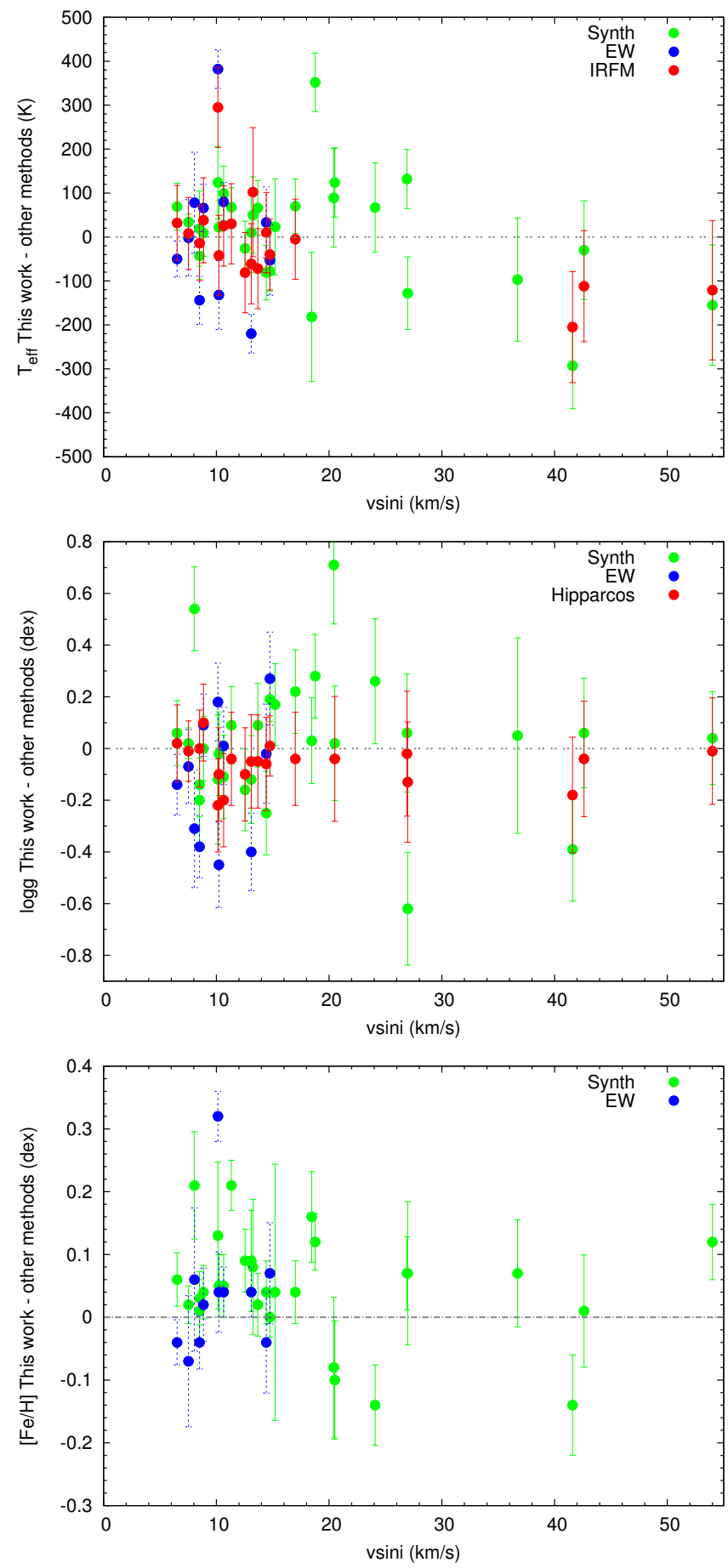

Fig. 7. Differences in temperature (top panel), surface gravity (middle panel), and metallicity (bottom panel) versus rotational velocity for moderate-to-fast rotators.

in Table 7. The stellar masses and radii are calculated using the calibration of Torres et al. (2010) with the corrections of Santos et al. (2013).

\subsection{Transit analysis}

From the literature we retrieved available photometric data for our stars with transiting planets. Our aim was to perform a homogeneous analysis of these objects using our redetermined stellar parameters to guess limb darkening coefficients and average stellar density. The limb darkening coefficients were linearly interpolated in the four dimensions of the new stellar parameters $\left(T_{\text {eff }}, \log g,[\mathrm{Fe} / \mathrm{H}]\right.$, and $\left.v_{\text {mac }}\right)$ from the tables of Claret $\&$ Bloemen (2011) to match our stellar parameter values. We also obtained the stellar density from the mass and radius as described in the previous section. Transit duration and transit depth were initially taken from the values quoted in the literature. The light curves were all folded with the period known from the literature, and out of transit measurements normalized to one.

Since some of the planets in our sample are in eccentric orbits, we adopted the expansion to the fourth order for the normalized projected distance of the planet with respect to the stellar center reported in Pál et al. (2010) and express it as a function of the stellar density $\left(\rho_{\star}\right)$ and the transit duration $\left(T_{\mathrm{d}}\right)$.

For each folded light curve, we fit a transiting planet model using the Mandel \& Agol (2002) model and the LevembergMarquardt algorithm (Press et al. 1992). For eccentric planets we adopted the values of the eccentricity and argument of periastron reported in the literature and add a Gaussian prior condition on both during our error analysis (see below), considering the reported uncertainties.

The uncertainties of the measurements were first expanded by the reduced $\chi^{2}$ of the fit. We accounted for correlated noise by creating a mock sample of the fit residuals (using the measurement uncertainties) and by comparing the scatter in the artificial and in the real light curves. The residuals were re-binned on increasing time intervals (up to $30 \mathrm{~min}$ ). If the ratio of the expected to the real scatter was found to be greater than one, we expanded the uncertainties further by this factor. Finally, we determined the distributions of the parameter best-fit values by bootstrapping the light curves and derived the mode of the resulting distributions and the 68.3 per cent confidence limits defined by the 15.85 th and the 84.15 th percentiles in the cumulative distributions.

The results are reported in Table 8. The photometric densities appear lower than the values implied by theoretical models. The discrepancy is greatest for the case of Kepler-410A where models predict $\rho_{\star} \sim 1 \mathrm{~g} \mathrm{~cm}^{-3}$, whereas the measured value is $0.0937_{-0.0052}^{+0.0070} \mathrm{~g} \mathrm{~cm}^{-3}$. The dilution caused by the contamination of a stellar companion (Kepler-410B) and the small size of the planet $\left(2.838 R_{\oplus}\right.$, Van Eylen et al. 2014) are the main reasons for the difference in the density derived from the transit fit. Considering the above, we have excluded this star from the comparison of the transit fit results.

\subsection{Discussion}

For stars with a transiting planet, it has been proposed that surface gravity is independently derived from the light curve with better precision than from spectroscopy (Seager \& Mallén-Ornelas 2003; Sozzetti et al. 2007). In Torres et al. (2012), it has been shown that $\log g$ derived using SME and the methodology of Valenti \& Fischer (2005) is systematically underestimated for hotter stars $\left(T_{\text {eff }}>6000 \mathrm{~K}\right)$ when compared with the $\log g$ from transit fits. According to the authors, constraining $\log g$ to the transit values, as presumably more reliable, leads to significant biases in the temperature and metallicity which consequently propagates to biases in stellar (and planetary) mass and radius.

From the planet hosts in our work, there are eight stars with transit data and available log $g$ from a light curve analysis. We therefore compare the $\log g$ derived from our spectroscopic analysis with the $\log g$ from the transit fits as taken from the 
Table 7. Spectroscopic parameters of planet hosts derived in this work and the literature values of the surface gravities from the transit light curve $\left(\log g_{\text {transit }}\right)$ for all transiting planet hosts in our sample.

\begin{tabular}{lcccccccc}
\hline \hline Star & $\begin{array}{c}T_{\text {eff }} \\
\mathrm{K}\end{array}$ & $\begin{array}{c}\log g \\
\mathrm{dex}\end{array}$ & $\begin{array}{c}{[\mathrm{Fe} / \mathrm{H}]} \\
\mathrm{dex}\end{array}$ & $\begin{array}{c}v \sin i \\
\mathrm{~km} \mathrm{~s}^{-1}\end{array}$ & $\begin{array}{c}\log g_{\text {transit }} \\
\text { dex }\end{array}$ & $\begin{array}{c}\text { Ref. } \\
\text { Mass } \\
M_{\odot}\end{array}$ & $\begin{array}{c}\text { Radius } \\
R_{\odot}\end{array}$ \\
\hline HAT-P-23 & $5924 \pm 30$ & $4.28 \pm 0.11$ & $0.16 \pm 0.03$ & $8.50 \pm 0.22$ & $4.33 \pm 0.05$ & $(1)$ & $1.13 \pm 0.05$ & $1.29 \pm 0.05$ \\
Kepler-410A & $6375 \pm 44$ & $4.25 \pm 0.15$ & $0.09 \pm 0.04$ & $13.24 \pm 0.29$ & $4.13 \pm 0.11$ & $(2)$ & $1.30 \pm 0.07$ & $1.41 \pm 0.07$ \\
CoRoT-3 & $6558 \pm 44$ & $4.25 \pm 0.15$ & $0.14 \pm 0.04$ & $18.46 \pm 0.29$ & $4.25 \pm 0.07$ & $(3)$ & $1.41 \pm 0.08$ & $1.44 \pm 0.08$ \\
XO-3 & $6781 \pm 44$ & $4.23 \pm 0.15$ & $-0.08 \pm 0.04$ & $18.77 \pm 0.29$ & $4.24 \pm 0.04$ & $(4)$ & $1.41 \pm 0.08$ & $1.49 \pm 0.08$ \\
HAT-P-41 & $6479 \pm 51$ & $4.39 \pm 0.22$ & $0.13 \pm 0.05$ & $20.11 \pm 1.34$ & $4.22 \pm 0.07$ & $(5)$ & $1.28 \pm 0.09$ & $1.19 \pm 0.08$ \\
HAT-P-2 & $6414 \pm 51$ & $4.18 \pm 0.22$ & $0.04 \pm 0.05$ & $20.50 \pm 1.34$ & $4.14 \pm 0.03$ & $(6)$ & $1.34 \pm 0.09$ & $1.54 \pm 0.12$ \\
HAT-P-34 & $6509 \pm 51$ & $4.24 \pm 0.22$ & $0.08 \pm 0.05$ & $24.08 \pm 1.34$ & $4.21 \pm 0.06$ & $(7)$ & $1.36 \pm 0.10$ & $1.45 \pm 0.11$ \\
HD 8673 & $6472 \pm 51$ & $4.27 \pm 0.22$ & $0.14 \pm 0.05$ & $26.91 \pm 1.34$ & - & - & $1.35 \pm 0.10$ & $1.39 \pm 0.10$ \\
CoRoT-11 & $6343 \pm 72$ & $4.27 \pm 0.30$ & $0.04 \pm 0.03$ & $36.72 \pm 1.34$ & $4.26 \pm 0.06$ & $(8)$ & $1.56 \pm 0.10$ & $1.36 \pm 0.13$ \\
30 Ari B & $6284 \pm 98$ & $4.35 \pm 0.20$ & $0.12 \pm 0.08$ & $42.61 \pm 1.82$ & - & - & $1.22 \pm 0.08$ & $1.23 \pm 0.07$ \\
\hline
\end{tabular}

References. (1) Bakos et al. (2011); (2) Van Eylen et al. (2014); (3) Deleuil et al. (2008); (4) Johns-Krull et al. (2008); (5) Hartman et al. (2012); (6) Pál et al. (2010); (7) Bakos et al. (2012); (8) Gandolfi et al. (2010).
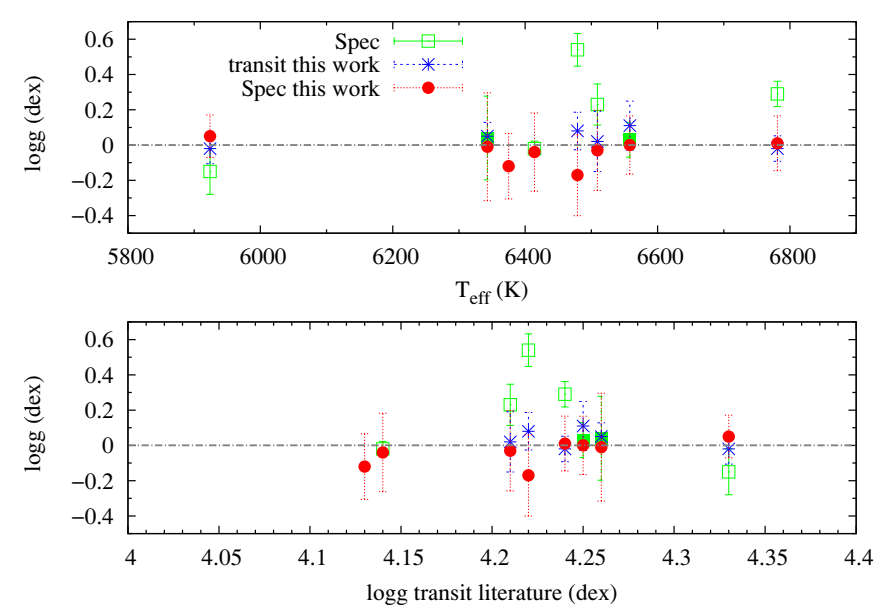

Fig. 8. Differences in surface gravity derived from a transit light curve analysis minus other methods from Table 7 . Circles correspond to the comparison of $\log g$ derived in this work. Squares correspond to the methodology applied by Valenti \& Fischer (2005) and filled squares to other spectral synthesis methods. Asterisks show the comparison between $\log g$ derived from the light curve analysis of the literature and of this work.

Table 8. Transit fit parameters.

\begin{tabular}{|c|c|c|c|c|}
\hline Name & $\bar{R} R_{\mathrm{p}} / R_{\star}$ & $\begin{array}{c}T_{\mathrm{d}} \\
\text { days }\end{array}$ & $\begin{array}{c}\rho_{\star} \\
\mathrm{g} \mathrm{cm}^{-3}\end{array}$ & $g_{1}$ \\
\hline HAT & $0.1209_{-0.0011}^{+0.0015}$ & $0.0822_{-0.0008}^{+0.0005}$ & $0.976_{-0.102}^{+0.068}$ & $0.281_{-0.056}^{+0.037}$ \\
\hline HAT & $0.0842_{-}^{+}$ & $0.1323_{-}^{+}$ & $0.505_{-c}^{+}$ & $0.037_{-0.019}^{+0.111}$ \\
\hline HAT-P-4 & 0.1049 & $0.1523^{+}$ & $0.452^{+}$ & 0.21 \\
\hline XO-3 & $0.0915_{-0}^{+0}$ & $0.1043_{-0}^{+0 .}$ & $0.649_{-0.060}^{+0.064}$ & $0.343_{-0.090}^{+0.034}$ \\
\hline CoRoT-3 & $0.0641_{-0 .}^{+0.8}$ & $0.1410_{-0.0008}^{+0.0010}$ & $0.431_{-0.055}^{+0.074}$ & $0.202^{+0.041}$ \\
\hline CoRoT-11 & $0.0999_{-0.0005}^{+0.0006}$ & $0.0799_{-0.0012}^{+0.0010}$ & $0.581_{-0.023}^{+0.035}$ & $0.347_{-0.092}^{+0.062}$ \\
\hline
\end{tabular}

literature (Fig. 8). The differences of this comparison are very small ( $\Delta \log g=-0.04$ with $\sigma=0.07 \mathrm{dex})$. On the other hand, a comparison between the log $g$ from the transit light curve and the $\log g$ using only the unconstrained methodology of Valenti $\&$ Fischer (2005) shows difference of $0.18(\sigma=0.27)$ dex for five stars with available measurements. For completeness, we also plot the $\log g$ from our light curve analysis of the previous section, using the stellar density and mass.
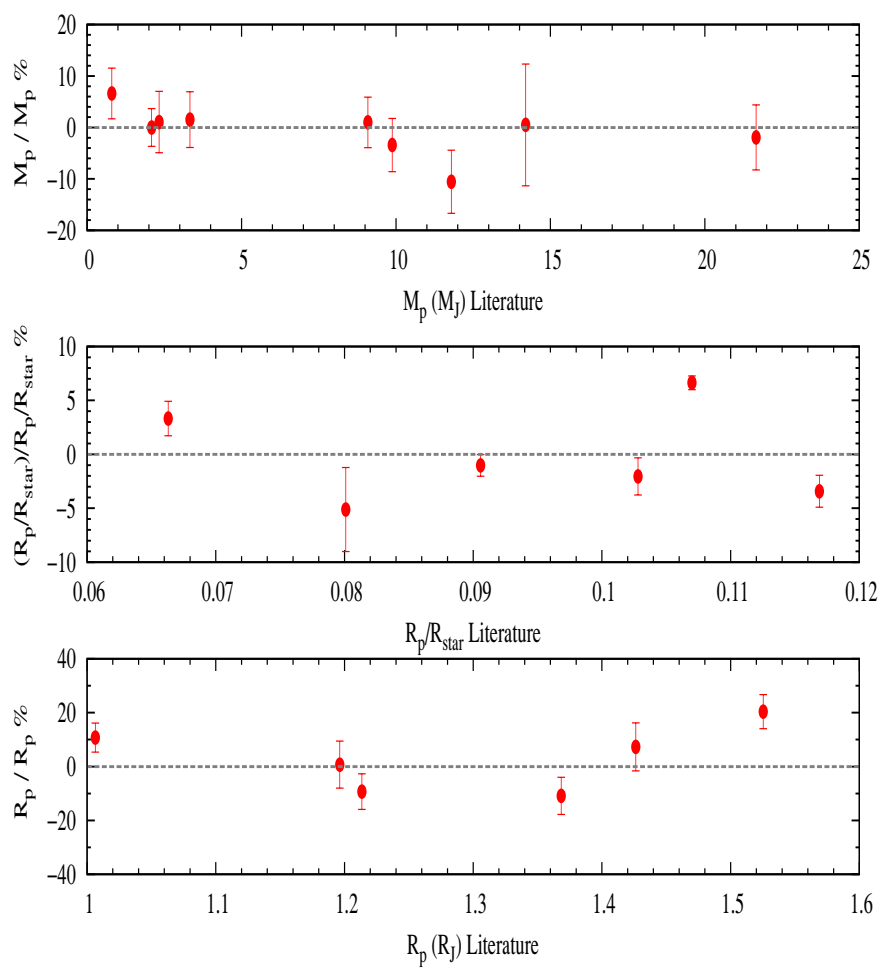

Fig. 9. Comparison between the literature data of planetary mass, the radii ratio $\left(R_{\mathrm{p}} / R_{\mathrm{star}}\right)$, and planetary radius, and this work in absolute units.

Even though the number of stars for this comparison is very small, these results suggest that fixing $\log g$ to the transit value is not required with the analysis of this work, avoiding the biases that are described in Torres et al. (2012). The different approach we adopt in this work, mainly because of the different line list, shows that we obtain a better estimate on surface gravity. However, since our sample is small and limited to hotter stars, further investigation is advised to check whether following the unconstrained approach is the optimal strategy. The unconstrained analysis is also suggested in Gómez Maqueo Chew et al. (2013) as preferable, after analyzing the transit host WASP-13 with SME but following different methodology (line list, initial parameters, convergence criteria, fixed parameters) from Valenti $\&$ Fischer (2005). 


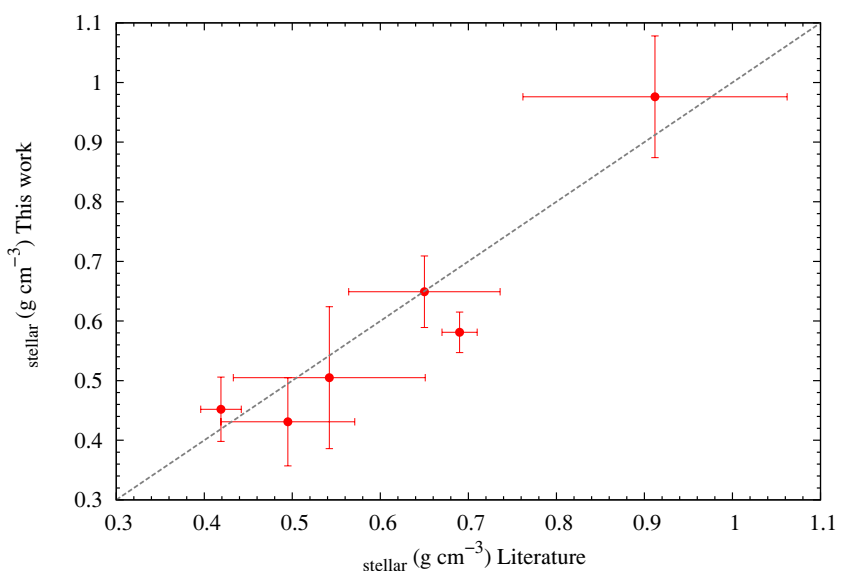

Fig. 10. Comparison between stellar density derived from the transit light curve analysis and literature data.

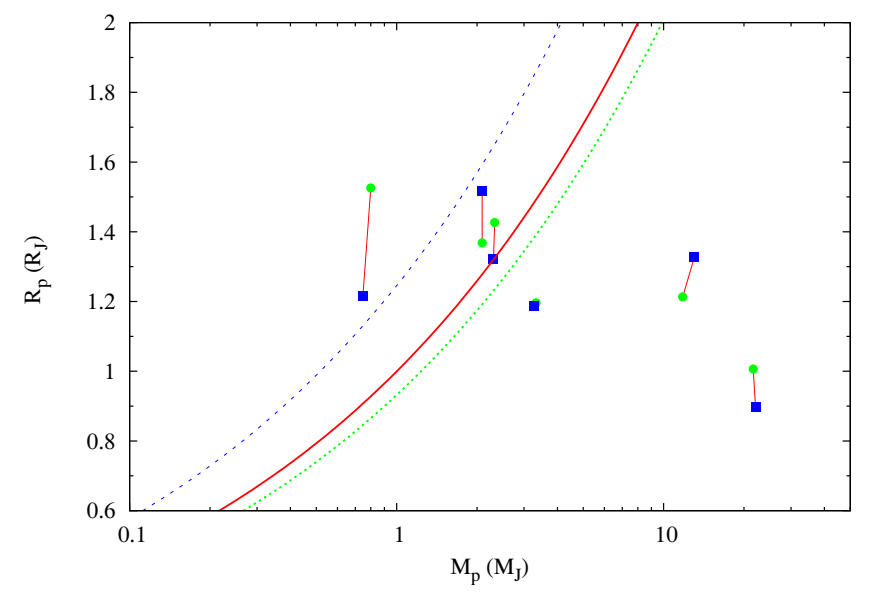

Fig. 11. Blue squares represent planetary mass and radius derived in this work in comparison with literature values (green circles). Characteristic isodensity curves are plotted for Saturn (dashed), Jupiter (solid), and Neptune (dotted).

We explored how the literature values of planetary mass and radius are affected with the new stellar parameters. From our analysis we find that the dispersion between the planetary mass derived with our stellar parameters and the literature is $4 \%$ (Fig. 9, top panel). The planet-to-star radius ratio derived from the transit light curve shows the same dispersion of $4 \%$ (Fig. 9, middle panel). This consistency with the literature values confirms the accuracy of the transit light curve analysis for deriving the planet-to-star radius ratio. The planetary radius is calculated from this ratio and the stellar radius that is inferred from our spectroscopic values. The comparison of the planetary radius with the literature values shows the highest dispersion of $14 \%$ (Fig. 9, bottom panel). Since we have shown the consistency of the planet-to-star radius ratio, the main source of uncertainty in the derivation of planetary radius is the calculation of the stellar value.

We also compared the stellar density derived from the transit analysis with the respective ones from the literature (Fig. 10). In Fig. 11, we show the new mass and radius from this work in comparison with the literature values. Planetary radius shows higher discrepancies mainly because of the uncertainties in the stellar radius calculations.

\section{Conclusions}

In this paper we have introduced a new approach to deriving the fundamental stellar parameters for FGK dwarfs using the spectral synthesis technique. In particular, we focused on stars with moderate-to-high rotational velocities. Such stars could be transiting planet hosts because they show high dispersion in their rotational velocities. The determination of their stellar parameters is very important for planetary studies.

The key to our method is the selection of the line list that mainly contains iron lines from previous work. This line list was tested primarily for stars with low rotational velocities. The comparison in temperature between the EW method and this work for our test sample is in good agreement even though high temperatures $\left(T_{\text {eff }}>6000 \mathrm{~K}\right)$ are underestimated by the synthesis technique. The metallicity is in excellent agreement with our test sample, and the surface gravity shows an offset of -0.19 dex.

Our method was applied to reference stars that are convolved with a set of rotational profiles up to $v \sin i=50 \mathrm{~km} \mathrm{~s}^{-1}$. The spectrum of each reference star has been broadened with different $v \sin i$ values, and for these spectra we calculated the stellar parameters, which are compared with the initial unbroadened spectrum in order to check their consistency for high $v \sin i$. The results show that even for high $v \sin i$, the differences from the ones without broadening are small and on the same scale as the errors.

As a final test we calculated stellar parameters for a sample of stars with high $v \sin i$ and compare with other spectral synthesis methodologies, the EW method (when possible), and the IRFM. The comparison shows very good agreement with all methods with the same dispersion in the mean differences of the parameters as the slowly rotating stars.

We also applied our method to ten planet hosts with moderate-to-fast rotation so their stellar parameters were derived in a uniform way. A new analysis has been conducted to the light curves for the stars that had available photometric observations using our results. From the combination of spectroscopic parameters and the ones derived from the transit fits (namely stellar density), we calculated the surface gravity. The comparison with spectroscopic derivations suggests that fixing $\log g$ to the transit value is not required using our method. In addition, we presented the difference in the planetary mass and radius (expressed as a percentage) with the literature values. The planetary masses agree very well with the literature values. The dispersion in the radii of the planets is higher owing to larger errors in the estimation of the stellar radius.

The study of planet hosts with higher rotational velocities is essential because they expand the planet sample around stars of earlier types (F- and A-types) that are more massive than the Sun. Precise stellar parameters for these stars are necessary for studying the frequency of planets around intermediate mass stars and exploring their planet formation mechanisms. Additionally, precise (and if possible accurate) stellar parameters are essential for a detailed characterization of the planets to be discovered by the upcoming high precision transit missions, such as CHEOPS, TESS, and PLATO 2.0.

Acknowledgements. This work is supported by the European Research Council/European Community under the FP7 through Starting Grant agreement number 239953. N.C.S. also acknowledges the support from Fundação para a Ciência e a Tecnologia (FCT) through program Ciência 2007 funded by FCT/MCTES (Portugal) and POPH/FSE (EC), and in the form of grant reference PTDC/CTE-AST/098528/2008. S.G.S, E.D.M, and V.Zh.A. acknowledge the support from the Fundação para a Ciência e Tecnologia, FCT (Portugal) in the form of the fellowships SFRH/BPD/47611/2008, SFRH/BPD/76606/2011, and SFRH/BPD/70574/2010. G.I. acknowledges financial support from the 
Spanish Ministry project MINECO AYA2011-29060. This research made use of the SIMBAD database operated at the CDS, Strasbourg, France, and the Vienna Atomic Line Database operated at Uppsala University, the Institute of Astronomy RAS in Moscow, and the University of Vienna. We thank the authors of SME for making their code public. The Narval observations were conducted under the OPTICON access program. OPTICON has received research funding from the European Community's Sixth Framework Program under contract number RII3-CT-001566.

\section{References}

Adibekyan, V. Z., Delgado Mena, E., Sousa, S. G., et al. 2012a, A\&A, 547, A36

Adibekyan, V. Z., Santos, N. C., Sousa, S. G., et al. 2012b, A\&A, 543, A89

Ammler-von Eiff, M., Santos, N. C., Sousa, S. G., et al. 2009, A\&A, 507, 523

Baglin, A., Auvergne, M., Boisnard, L., et al. 2006, in 36th COSPAR Scientific Assembly, COSPAR Meeting, 36, 3749

Bakos, G., Noyes, R. W., Kovcs, G., et al. 2004, PASP, 116, 266

Bakos, G. Á., Hartman, J., Torres, G., et al. 2011, ApJ, 742, 116

Bakos, G. Á., Hartman, J. D., Torres, G., et al. 2012, AJ, 144, 19

Bensby, T., Feltzing, S., \& Oey, M. S. 2014, A\&A, 562, A71

Bessell, M. S., Castelli, F., \& Plez, B. 1998, A\&A, 333, 231

Borucki, W. J., Koch, D., Basri, G., et al. 2010, Science, 327, 977

Bouchy, F., Pepe, F., \& Queloz, D. 2001, A\&A, 374, 733

Bruntt, H., Bikmaev, I. F., Catala, C., et al. 2004, A\&A, 425, 683

Bruntt, H., Bedding, T. R., Quirion, P.-O., et al. 2010, MNRAS, 405, 1907

Bruntt, H., Basu, S., Smalley, B., et al. 2012, MNRAS, 423, 122

Burrows, A., Guillot, T., Hubbard, W. B., et al. 2000, ApJ, 534, L97

Casagrande, L., Schönrich, R., Asplund, M., et al. 2011, A\&A, 530, A138

Claret, A., \& Bloemen, S. 2011, A\&A, 529, A75

da Silva, L., Girardi, L., Pasquini, L., et al. 2006, A\&A, 458, 609

Deleuil, M., Deeg, H. J., Alonso, R., et al. 2008, A\&A, 491, 889

Everett, M. E., Howell, S. B., Silva, D. R., \& Szkody, P. 2013, ApJ, 771, 107

Flower, P. J. 1996, ApJ, 469, 355

Gandolfi, D., Hébrard, G., Alonso, R., et al. 2010, A\&A, 524, A55

Girardi, L., Bertelli, G., Bressan, A., et al. 2002, A\&A, 391, 195

Gómez Maqueo Chew, Y., Faedi, F., Cargile, P., et al. 2013, ApJ, 768, 79

Gray, D. F. 1984, ApJ, 281, 719

Gray, R. O., Corbally, C. J., Garrison, R. F., et al. 2006, AJ, 132, 161

Hartman, J. D., Bakos, G. Á., Béky, B., et al. 2012, AJ, 144, 139

Hebb, L., Collier-Cameron, A., Loeillet, B., et al. 2009, ApJ, 693, 1920

Hekker, S., \& Meléndez, J. 2007, A\&A, 475, 1003

Hubeny, I., Lanz, T., \& Jeffery, C. S. 1994

Huber, D., Chaplin, W. J., Christensen-Dalsgaard, J., et al. 2013, ApJ, 767, 127

Johns-Krull, C. M., McCullough, P. R., Burke, C. J., et al. 2008, ApJ, 677, 657

Kupka, F., Piskunov, N., Ryabchikova, T. A., Stempels, H. C., \& Weiss, W. W. 1999, A\&AS, 138, 119
Kurucz, R. 1993, ATLAS9 Stellar Atmosphere Programs and $2 \mathrm{~km} \mathrm{~s}^{-1}$ grid. Kurucz CD-ROM No. 13 (Cambridge, Mass.: Smithsonian Astrophysical Observatory), 13

Kurucz, R. L., Furenlid, I., Brault, J., \& Testerman, L. 1984, Solar flux atlas from 296 to $1300 \mathrm{~nm}$

Malavolta, L., Sneden, C., Piotto, G., et al. 2014, AJ, 147, 25

Mandel, K., \& Agol, E. 2002, ApJ, 580, L171

Molenda-Żakowicz, J., Sousa, S. G., Frasca, A., et al. 2013, MNRAS, 434, 1422

Montalto, M., Gregorio, J., Boué, G., et al. 2012, MNRAS, 427, 2757

Mordasini, C., Alibert, Y., Georgy, C., et al. 2012, A\&A, 547, A112

Mortier, A., Santos, N. C., Sousa, S. G., et al. 2013a, A\&A, 557, A70

Mortier, A., Santos, N. C., Sousa, S. G., et al. 2013b, A\&A, 558, A106

Nielsen, M. B., Gizon, L., Schunker, H., \& Karoff, C. 2013, A\&A, 557, L10

Nissen, P. E. 1981, A\&A, 97, 145

Noyes, R. W., Bakos, G. Á., Torres, G., et al. 2008, ApJ, 673, L79

Pál, A., Bakos, G. Á., Torres, G., et al. 2010, MNRAS, 401, 2665

Piskunov, N. E., Kupka, F., Ryabchikova, T. A., Weiss, W. W., \& Jeffery, C. S. 1995, A\&AS, 112, 525

Pollacco, D. L., Skillen, I., Cameron, A. C., et al. 2006, PASP, 118, 1407

Pollacco, D., Skillen, I., Collier Cameron, A., et al. 2008, MNRAS, 385, 1576

Press, W. H., Teukolsky, S. A., Vetterling, W. T., \& Flannery, B. P. 1992, Numerical recipes in FORTRAN. The art of scientific computing

Prugniel, P., Vauglin, I., \& Koleva, M. 2011, A\&A, 531, A165

Ramírez, I., Allende Prieto, C., \& Lambert, D. L. 2013, ApJ, 764, 78

Recio-Blanco, A., Bijaoui, A., \& de Laverny, P. 2006, MNRAS, 370, 141

Saar, S. H., \& Osten, R. A. 1997, MNRAS, 284, 803

Santos, N. C., Israelian, G., \& Mayor, M. 2004, A\&A, 415, 1153

Santos, N. C., Israelian, G., Mayor, M., et al. 2005, A\&A, 437, 1127

Santos, N. C., Sousa, S. G., Mortier, A., et al. 2013, A\&A, 556, A150

Seager, S., \& Mallén-Ornelas, G. 2003, ApJ, 585, 1038

Sousa, S. G., Santos, N. C., Israelian, G., Mayor, M., \& Udry, S. 2011, A\&A, 533, A141

Sousa, S. G., Santos, N. C., Mayor, M., et al. 2008, A\&A, 487, 373

Sozzetti, A., Torres, G., Charbonneau, D., et al. 2007, ApJ, 664, 1190

Takeda, Y. 2007, PASJ, 59, 335

Torres, G. 2010, AJ, 140, 1158

Torres, G., Winn, J. N., \& Holman, M. J. 2008, ApJ, 677, 1324

Torres, G., Andersen, J., \& Giménez, A. 2010, A\&ARv, 18, 67

Torres, G., Fischer, D. A., Sozzetti, A., et al. 2012, ApJ, 757, 161

Tsantaki, M., Sousa, S. G., Adibekyan, V. Z., et al. 2013, A\&A, 555, A150

Valenti, J. A., \& Fischer, D. A. 2005, ApJS, 159, 141

Valenti, J. A., \& Piskunov, N. 1996, A\&AS, 118, 595

Valenti, J. A., Piskunov, N., \& Johns-Krull, C. M. 1998, ApJ, 498, 851

Van Eylen, V., Lund, M. N., Silva Aguirre, V., et al. 2014, ApJ, 782, 14

van Leeuwen, F. 2007, A\&A, 474, 653

Verner, G. A., Chaplin, W. J., Basu, S., et al. 2011, ApJ, 738, L28

Viana Almeida, P., Santos, N. C., Melo, C., et al. 2009, A\&A, 501, 965 
M. Tsantaki et al.: Spectroscopic parameters for solar-type starswith moderate-to-high rotation

\section{Appendix A}

Table A.1. Results of the comparison between this work and the EW method.

\begin{tabular}{|c|c|c|c|c|c|c|c|}
\hline \multirow[b]{2}{*}{ Star } & \multicolumn{4}{|c|}{ This work } & \multicolumn{3}{|c|}{$E W$ method } \\
\hline & $\begin{array}{l}T_{\text {eff }} \\
(\mathrm{K})\end{array}$ & $\begin{array}{l}\log g \\
(\operatorname{dex})\end{array}$ & $\begin{array}{c}{[\mathrm{Fe} / \mathrm{H}]} \\
(\mathrm{dex})\end{array}$ & $\begin{array}{c}v \sin i \\
\left(\mathrm{~km} \mathrm{~s}^{-1}\right)\end{array}$ & $\begin{array}{l}T_{\text {eff }} \\
(\mathrm{K})\end{array}$ & $\begin{array}{l}\log g \\
(\operatorname{dex})\end{array}$ & $\begin{array}{c}{[\mathrm{Fe} / \mathrm{H}]} \\
(\mathrm{dex})\end{array}$ \\
\hline \multicolumn{8}{|c|}{ Dwarf stars } \\
\hline CoRoT-2 & $5620 \pm 18$ & $4.66 \pm 0.06$ & $-0.03 \pm 0.03$ & 9.97 & $5697 \pm 97$ & $4.73 \pm 0.17$ & $-0.09 \pm 0.07$ \\
\hline CoRoT-10 & $4921 \pm 25$ & $4.09 \pm 0.09$ & $0.15 \pm 0.03$ & 2.19 & $5025 \pm 155$ & $4.47 \pm 0.31$ & $0.06 \pm 0.09$ \\
\hline CoRoT-4 & $6164 \pm 30$ & $4.34 \pm 0.11$ & $0.15 \pm 0.03$ & 7.03 & $6344 \pm 93$ & $4.82 \pm 0.11$ & $0.15 \pm 0.06$ \\
\hline CoRoT-5 & $6254 \pm 30$ & $4.41 \pm 0.11$ & $0.04 \pm 0.03$ & 1.43 & $6240 \pm 70$ & $4.46 \pm 0.11$ & $0.04 \pm 0.05$ \\
\hline HD 101930 & $5083 \pm 18$ & $4.15 \pm 0.06$ & $0.10 \pm 0.03$ & 0.10 & $5083 \pm 63$ & $4.35 \pm 0.13$ & $0.16 \pm 0.04$ \\
\hline HD 102365 & $5588 \pm 18$ & $4.07 \pm 0.06$ & $-0.30 \pm 0.03$ & 0.10 & $5616 \pm 41$ & $4.40 \pm 0.06$ & $-0.28 \pm 0.03$ \\
\hline HD 103774 & $6582 \pm 30$ & $4.47 \pm 0.11$ & $0.27 \pm 0.03$ & 8.93 & $6732 \pm 56$ & $4.81 \pm 0.06$ & $0.29 \pm 0.03$ \\
\hline HD 1237 & $5588 \pm 18$ & $4.58 \pm 0.06$ & $0.11 \pm 0.03$ & 4.62 & $5489 \pm 40$ & $4.46 \pm 0.11$ & $0.06 \pm 0.03$ \\
\hline HD 134060 & $5914 \pm 18$ & $4.28 \pm 0.06$ & $0.09 \pm 0.03$ & 1.44 & $5940 \pm 18$ & $4.42 \pm 0.03$ & $0.12 \pm 0.01$ \\
\hline HD 1388 & $5967 \pm 18$ & $4.38 \pm 0.06$ & $0.00 \pm 0.03$ & 1.27 & $5970 \pm 15$ & $4.42 \pm 0.05$ & $0.00 \pm 0.01$ \\
\hline HD 148156 & $6212 \pm 30$ & $4.40 \pm 0.11$ & $0.23 \pm 0.03$ & 5.73 & $6251 \pm 25$ & $4.51 \pm 0.05$ & $0.25 \pm 0.02$ \\
\hline HD 162020 & $4798 \pm 25$ & $4.14 \pm 0.09$ & $-0.14 \pm 0.03$ & 1.46 & $4723 \pm 71$ & $4.31 \pm 0.18$ & $-0.10 \pm 0.03$ \\
\hline HD 20852 & $6675 \pm 30$ & $4.12 \pm 0.11$ & $-0.37 \pm 0.03$ & 7.06 & $6813 \pm 92$ & $4.76 \pm 0.12$ & $-0.35 \pm 0.06$ \\
\hline HD 20868 & $4745 \pm 25$ & $4.02 \pm 0.09$ & $0.00 \pm 0.03$ & 0.46 & $4720 \pm 91$ & $4.24 \pm 0.47$ & $0.08 \pm 0.01$ \\
\hline HD 221287 & $6337 \pm 30$ & $4.43 \pm 0.06$ & $0.02 \pm 0.06$ & 3.92 & $6417 \pm 25$ & $4.60 \pm 0.10$ & $0.06 \pm 0.02$ \\
\hline HD 222237 & $4618 \pm 25$ & $3.92 \pm 0.09$ & $-0.50 \pm 0.03$ & 0.10 & $4722 \pm 55$ & $4.34 \pm 0.15$ & $-0.39 \pm 0.06$ \\
\hline HD 23079 & $5965 \pm 18$ & $4.28 \pm 0.06$ & $-0.13 \pm 0.03$ & 0.10 & $6009 \pm 14$ & $4.50 \pm 0.05$ & $-0.11 \pm 0.01$ \\
\hline HD 27894 & $4894 \pm 25$ & $4.08 \pm 0.09$ & $0.18 \pm 0.03$ & 0.87 & $4833 \pm 209$ & $4.30 \pm 0.48$ & $0.26 \pm 0.10$ \\
\hline HD 31527 & $5915 \pm 18$ & $4.40 \pm 0.06$ & $-0.17 \pm 0.03$ & 2.36 & $5917 \pm 13$ & $4.47 \pm 0.05$ & $-0.17 \pm 0.01$ \\
\hline HD 330075 & $4924 \pm 30$ & $4.03 \pm 0.09$ & $-0.04 \pm 0.03$ & 0.10 & $4958 \pm 52$ & $4.24 \pm 0.13$ & $0.05 \pm 0.03$ \\
\hline HD 361 & $5924 \pm 18$ & $4.48 \pm 0.06$ & $-0.10 \pm 0.03$ & 0.10 & $5888 \pm 14$ & $4.54 \pm 0.08$ & $-0.13 \pm 0.01$ \\
\hline HD 38283 & $5962 \pm 18$ & $4.14 \pm 0.06$ & $-0.15 \pm 0.03$ & 4.51 & $5980 \pm 24$ & $4.27 \pm 0.03$ & $-0.14 \pm 0.02$ \\
\hline HD 40307 & $4771 \pm 25$ & $4.10 \pm 0.09$ & $-0.42 \pm 0.03$ & 0.10 & $4774 \pm 77$ & $4.42 \pm 0.16$ & $-0.36 \pm 0.02$ \\
\hline HD 61421 & $6616 \pm 30$ & $4.09 \pm 0.11$ & $0.03 \pm 0.03$ & 4.40 & 6612 & 4.02 & -0.02 \\
\hline HD 63454 & $4833 \pm 25$ & $4.11 \pm 0.09$ & $0.04 \pm 0.03$ & 1.81 & $4756 \pm 77$ & $4.32 \pm 0.22$ & $0.13 \pm 0.05$ \\
\hline HD 750 & $5118 \pm 18$ & $4.34 \pm 0.06$ & $-0.29 \pm 0.03$ & 0.10 & $5069 \pm 32$ & $4.33 \pm 0.1$ & $-0.30 \pm 0.02$ \\
\hline HD 870 & $5379 \pm 18$ & $4.36 \pm 0.06$ & $-0.12 \pm 0.03$ & 0.10 & $5360 \pm 24$ & $4.40 \pm 0.08$ & $-0.12 \pm 0.02$ \\
\hline HD 93385 & $5987 \pm 18$ & $4.38 \pm 0.06$ & $0.02 \pm 0.03$ & 1.06 & $5989 \pm 17$ & $4.46 \pm 0.03$ & $0.03 \pm 0.01$ \\
\hline HD 967 & $5643 \pm 18$ & $4.38 \pm 0.06$ & $-0.59 \pm 0.03$ & 0.10 & $5595 \pm 18$ & $4.59 \pm 0.02$ & $-0.66 \pm 0.01$ \\
\hline OGLE-TR-113 & $4793 \pm 25$ & $4.25 \pm 0.09$ & $0.05 \pm 0.03$ & 5.02 & $4781 \pm 166$ & $4.31 \pm 0.41$ & $0.03 \pm 0.06$ \\
\hline WASP-29 & $4782 \pm 25$ & $4.13 \pm 0.09$ & $0.18 \pm 0.03$ & 0.10 & $5203 \pm 102$ & $4.93 \pm 0.21$ & $0.17 \pm 0.05$ \\
\hline WASP-15 & $6378 \pm 30$ & $4.24 \pm 0.11$ & $0.03 \pm 0.03$ & 5.13 & $6573 \pm 70$ & $4.79 \pm 0.08$ & $0.09 \pm 0.03$ \\
\hline WASP-16 & $5710 \pm 18$ & $4.23 \pm 0.06$ & $0.12 \pm 0.03$ & 0.47 & $5726 \pm 22$ & $4.34 \pm 0.05$ & $0.13 \pm 0.02$ \\
\hline WASP-17 & $6666 \pm 30$ & $4.26 \pm 0.06$ & $-0.04 \pm 0.03$ & 9.93 & $6794 \pm 83$ & $4.83 \pm 0.09$ & $-0.12 \pm 0.05$ \\
\hline WASP-2 & $5105 \pm 18$ & $3.97 \pm 0.06$ & $0.08 \pm 0.03$ & 2.90 & $5109 \pm 72$ & $4.33 \pm 0.14$ & $0.02 \pm 0.05$ \\
\hline WASP-23 & $5053 \pm 18$ & $4.20 \pm 0.06$ & $-0.02 \pm 0.03$ & 0.46 & $5046 \pm 99$ & $4.33 \pm 0.18$ & $0.05 \pm 0.06$ \\
\hline WASP-38 & $6247 \pm 30$ & $4.25 \pm 0.11$ & $0.06 \pm 0.03$ & 8.05 & $6436 \pm 60$ & $4.80 \pm 0.07$ & $0.06 \pm 0.04$ \\
\hline WASP-6 & $5447 \pm 18$ & $4.42 \pm 0.06$ & $-0.11 \pm 0.03$ & 0.10 & $5383 \pm 41$ & $4.52 \pm 0.06$ & $-0.14 \pm 0.03$ \\
\hline Sun & $5771 \pm 18$ & $4.42 \pm 0.06$ & $0.00 \pm 0.03$ & 2.57 & - & - & - \\
\hline \multicolumn{8}{|c|}{ Giant s } \\
\hline HD 148427 & $5018 \pm 25$ & $3.49 \pm 0.09$ & $0.01 \pm 0.03$ & 0.45 & $4962 \pm 45$ & $3.39 \pm 0.12$ & $0.03 \pm 0.03$ \\
\hline HD 175541 & $5097 \pm 18$ & $3.44 \pm 0.06$ & $-0.14 \pm 0.03$ & 2.45 & $5111 \pm 38$ & $3.56 \pm 0.08$ & $-0.11 \pm 0.03$ \\
\hline HD 27442 & $4852 \pm 25$ & $3.48 \pm 0.09$ & $0.23 \pm 0.03$ & 2.65 & $4781 \pm 76$ & $3.46 \pm 0.19$ & $0.33 \pm 0.05$ \\
\hline HD 62509 & $5007 \pm 25$ & $3.06 \pm 0.09$ & $0.21 \pm 0.03$ & 3.76 & $4935 \pm 49$ & $2.91 \pm 0.13$ & $0.09 \pm 0.04$ \\
\hline HD 88133 & $5330 \pm 18$ & $3.62 \pm 0.06$ & $0.20 \pm 0.03$ & 3.39 & $5438 \pm 34$ & $3.94 \pm 0.11$ & $0.33 \pm 0.05$ \\
\hline HD 142091 & $4898 \pm 25$ & $3.24 \pm 0.09$ & $0.05 \pm 0.03$ & 4.38 & $4876 \pm 46$ & $3.15 \pm 0.14$ & $0.13 \pm 0.03$ \\
\hline HD 188310 & $4799 \pm 18$ & $3.14 \pm 0.06$ & $-0.06 \pm 0.03$ & 5.28 & $4714 \pm 49$ & $2.53 \pm 0.11$ & $-0.27 \pm 0.04$ \\
\hline HD 163917 & $5107 \pm 18$ & $2.82 \pm 0.06$ & $0.33 \pm 0.03$ & 4.21 & $4967 \pm 61$ & $2.70 \pm 0.13$ & $0.14 \pm 0.05$ \\
\hline
\end{tabular}

Notes. The stars in boldface are analyzed in Sect. 4. 


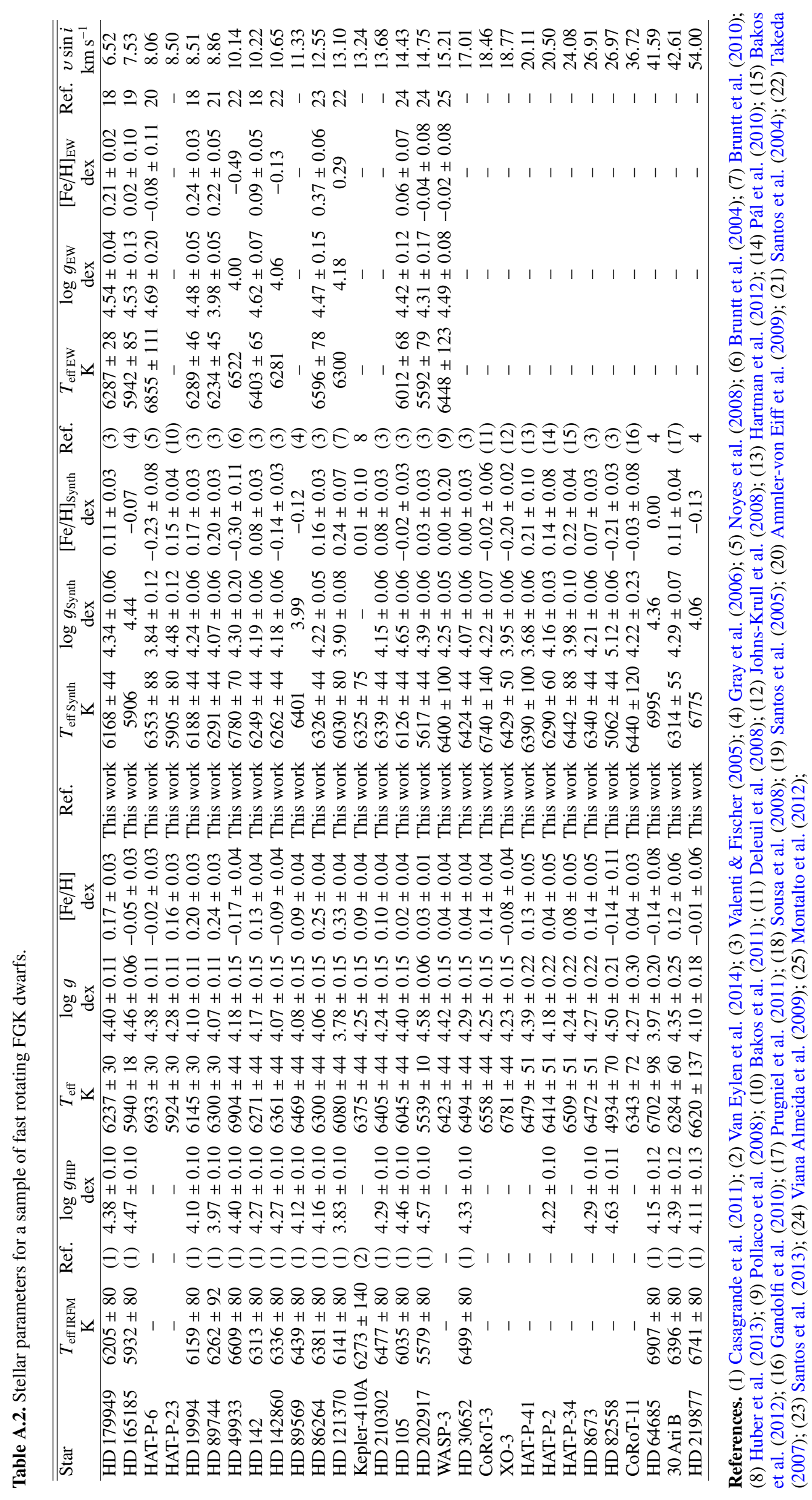

